\title{
NOVEL INTEGRATION OF CONDUCTIVE-INK CIRCUITRY \\ WITH A PAPER-BASED MICROFLUIDIC BATTERY \\ AS AN ALL-PRINTED SENSING PLATFORM
}

\author{
A Thesis \\ presented to \\ the Faculty of California Polytechnic State University, \\ San Luis Obispo
}

\author{
In Partial Fulfillment \\ of the Requirements for the Degree \\ Master of Science in Materials Engineering
}

by

Rishi A. Kripalani

December 2016 
(C) 2016

Rishi A. Kripalani

ALL RIGHTS RESERVED 


\title{
COMMITTEE MEMBERSHIP
}

TITLE: Novel Integration of Conductive-ink Circuitry with

a Paper-based Microfluidic Battery as an All-

printed Sensing Platform

\author{
AUTHOR: $\quad$ Rishi A. Kripalani
}

DATE SUBMITTED: December 2016

COMMITTE CHAIR: $\quad$ Linda Vanasupa, Ph.D.

Professor of Materials Engineering

COMMITTEE MEMBER: $\quad$ Katherine Chen, Ph.D.

Professor of Materials Engineering

COMMITTE MEMBER: $\quad$ Richard Savage, Ph.D.

Dean of Graduate Education 


\begin{abstract}
Novel Integration of Conductive-ink Circuitry with a Paper-based Microfluidic Battery as an All-printed Sensing Platform
\end{abstract}

\title{
Rishi A. Kripalani
}

The addition of powered components for active assays into paper-based analytical devices opens new opportunities for medical and environmental analysis in resourcelimited applications. Current battery designs within such devices have yet to adopt a ubiquitous circuitry material, necessitating investigation into printed circuitry for scalable platforms. In this study, a microfluidic battery was mated with silver-nanoparticle conductive ink to prototype an all-printed sensing platform. A multi-layer, two-cell device was fabricated, generating $200 \mu \mathrm{A}$ of direct electrical current at $2.5 \mathrm{~V}$ sustained for 16 minutes with a power loss of less than $0.1 \%$ through the printed circuitry. Printed circuitry traces exhibited resistivity of 75 to $21110^{-5} \Omega \cdot m$. Resistance of the printed traces increased upwards of $200 \%$ depending on fold angle and directionality. X-ray diffraction confirmed the presence of face-centered cubic silver after sintering printed traces for 30 minutes at $150^{\circ} \mathrm{C}$ in air. A conductivity threshold was mapped and an ink concentration of $0.636 \mu \mathrm{L} \cdot \mathrm{mm}^{-3}$ was identified as the lower limit for optimal electrical performance.

Keywords: silver nanoparticle ink, microfluidic battery, percolation threshold, paperbased analytical device, $\mu \mathrm{PAD}$, printed circuitry, $\mathrm{x}$-ray diffraction, resistivity 


\section{ACKNOWLEDGMENTS}

A special thanks is extended to the following's for their guidance and instruction:

Dr. Linda Vanasupa

Dr. Katherine Chen

Dr. Richard Savage

A special thanks is extended to the following for their collaboration:

Dr. Scott Phillips

Dr. Andres W. Martinez

Dr. David Clague

Dr. Nathaniel W. Martinez

Glenn Lee

Jeremy Dunn

Cory Hughes

A special thanks is extended to the following for their support and inspiration:

Lisa J. Rutherford

Alexandra Golobic

Anil \& Monica Kripalani 


\section{TABLE OF CONTENTS}

Page

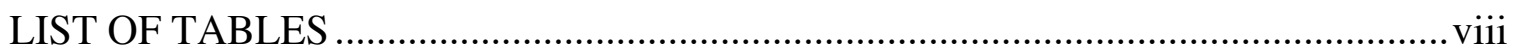

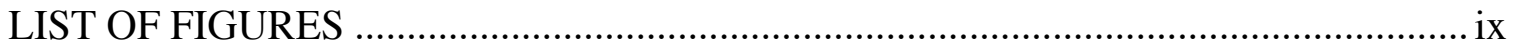

CHAPTER

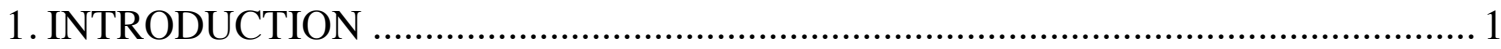

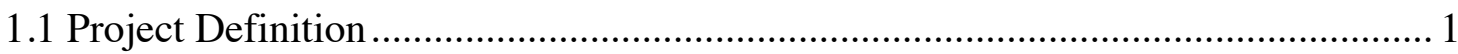

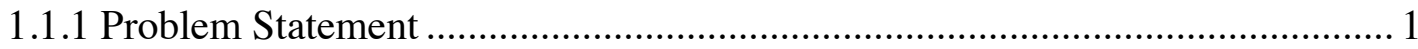

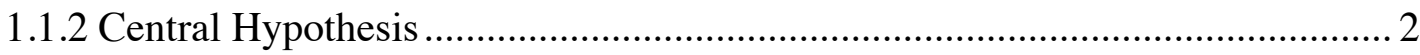

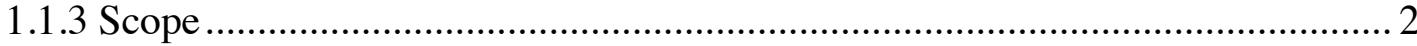

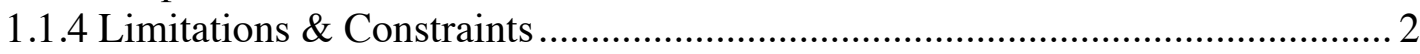

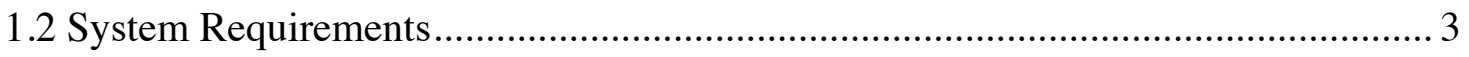

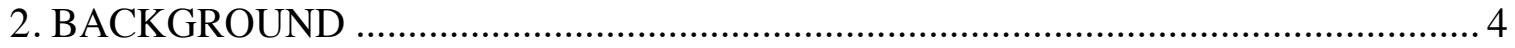

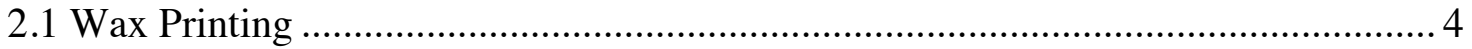

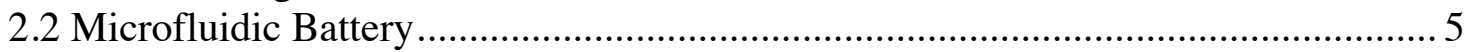

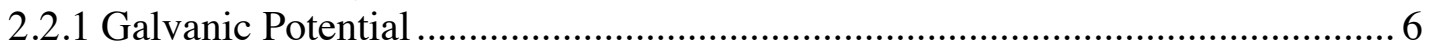

2.2.2 Layer Functions ………………….................................................. 9

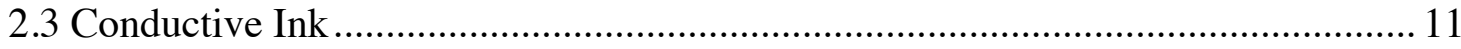

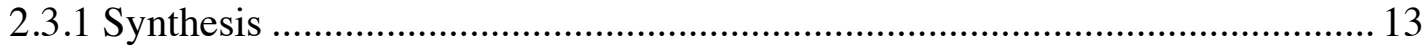

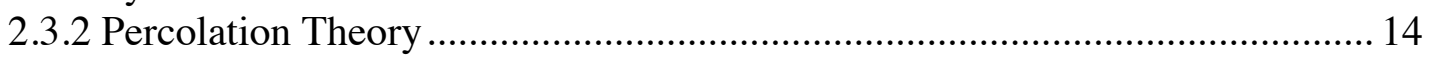

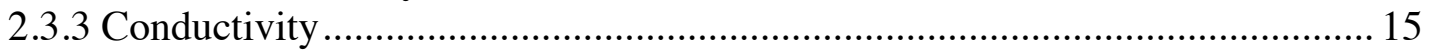

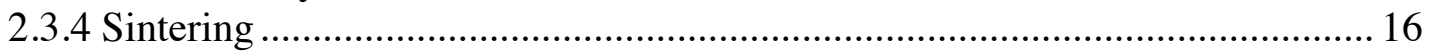

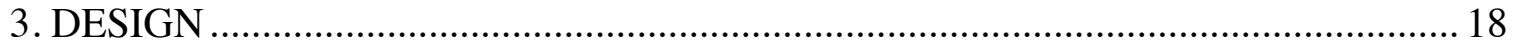

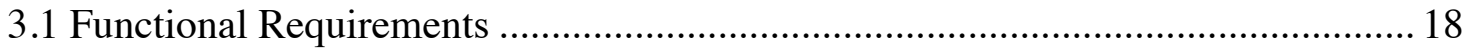

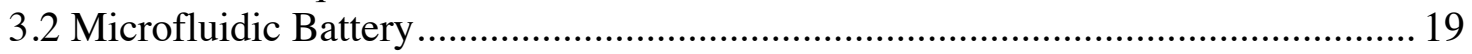

3.2.1 Design Adaptations ............................................................................ 19

3.2.2 Hydrophobic Layer Material................................................................... 20

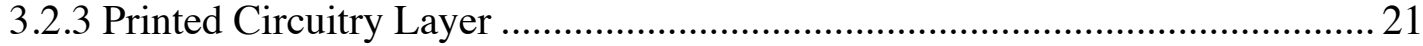

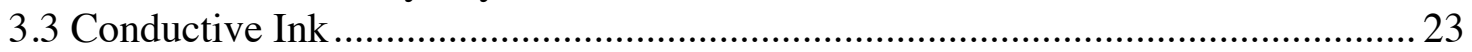

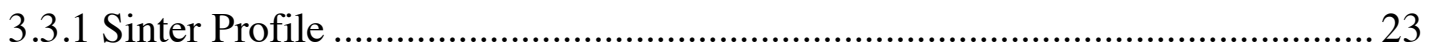

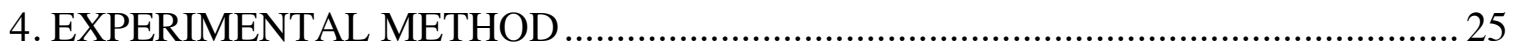

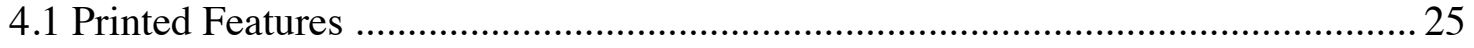

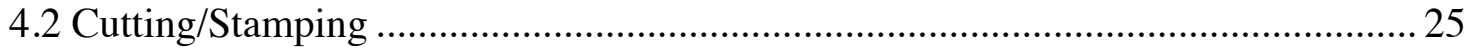

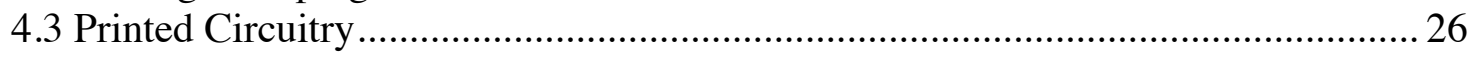

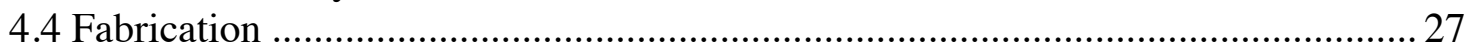

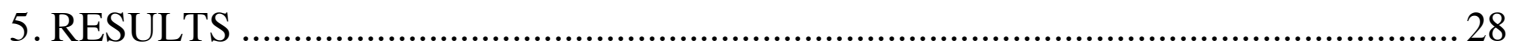

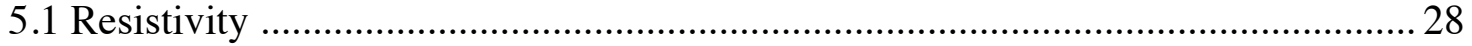

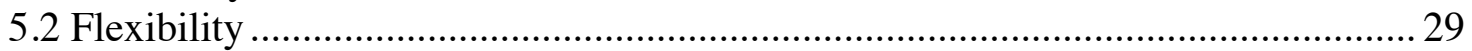

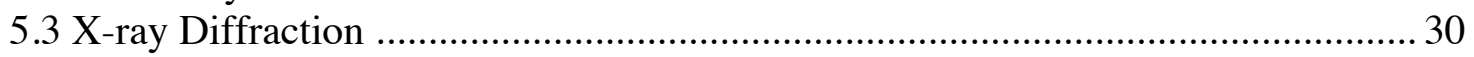

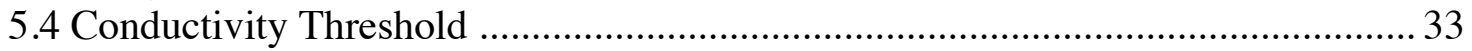

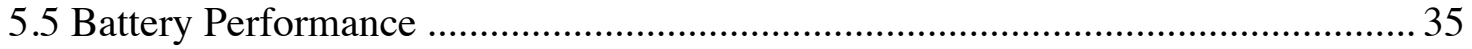

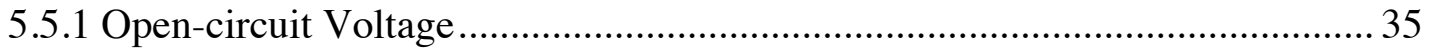

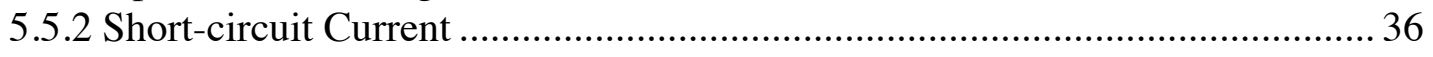

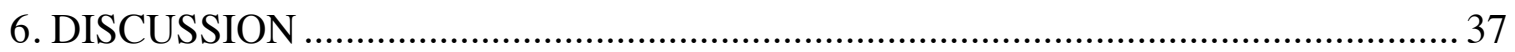




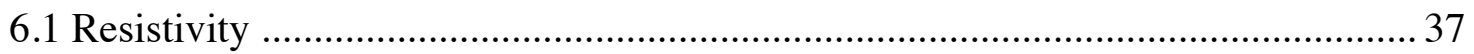

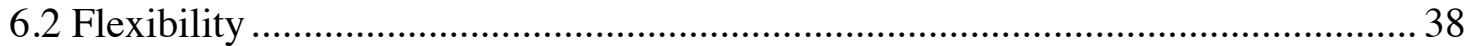

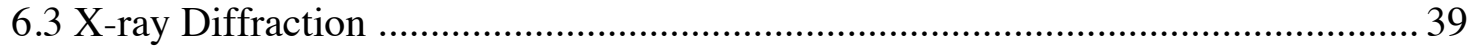

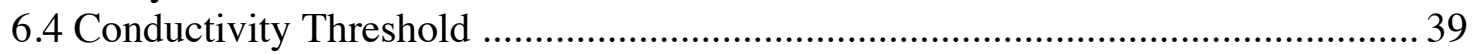

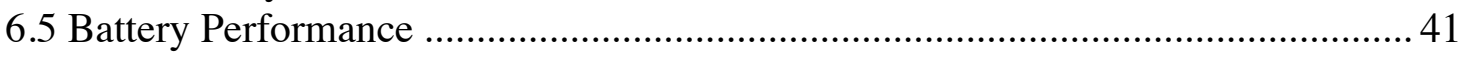

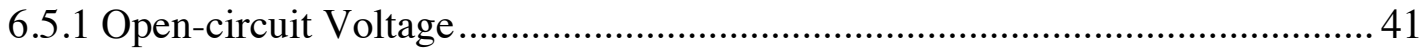

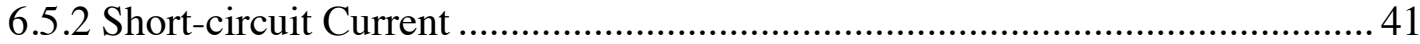

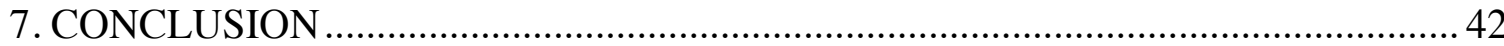

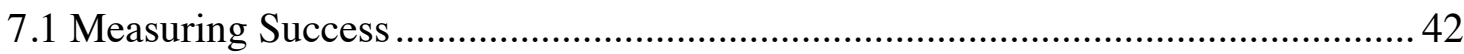

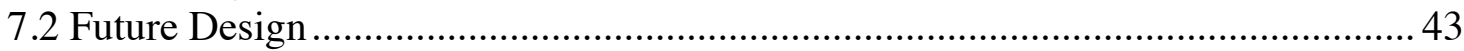

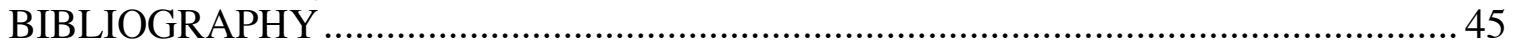

APPENDICES

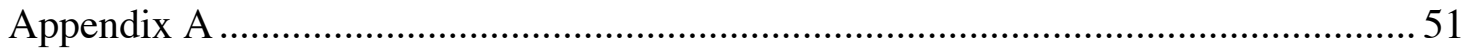

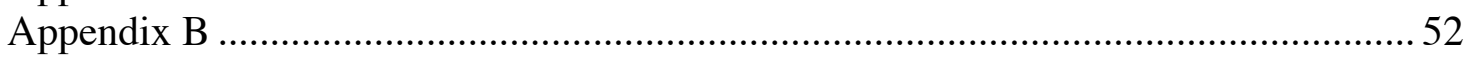

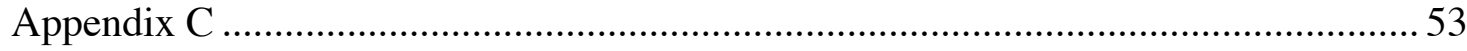

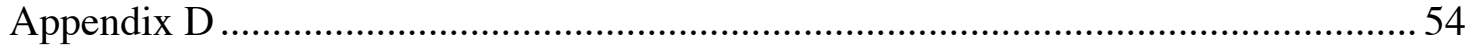

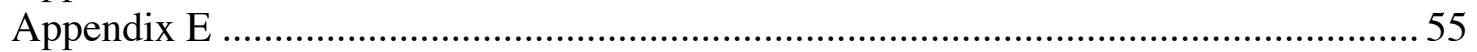

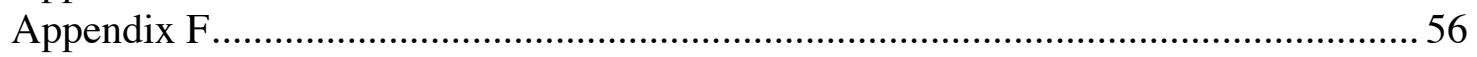

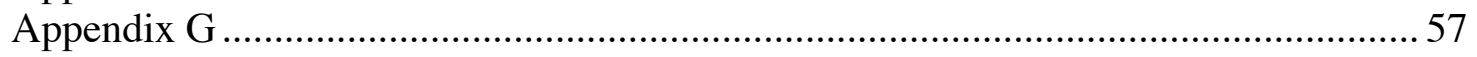




\section{LIST OF TABLES}

Table

Page

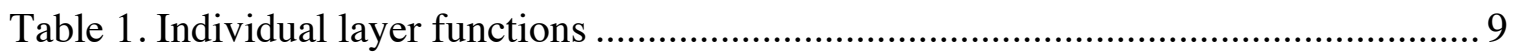

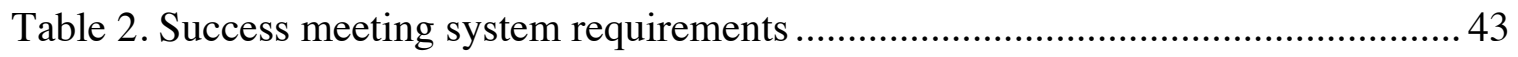

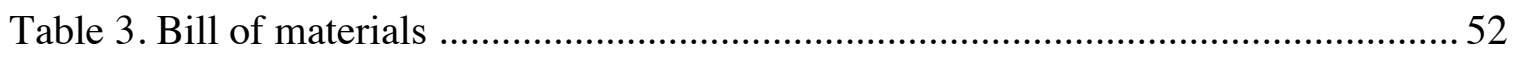

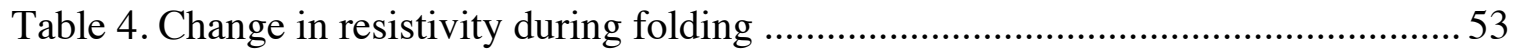

Table 5. Resistivity using two-factor sinter matrix ................................................ 54

Table 6. Resistivity using three-factor sinter matrix ................................................. 55

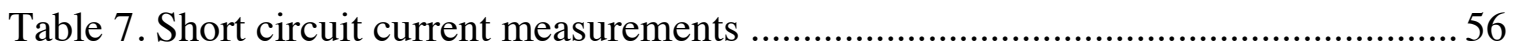

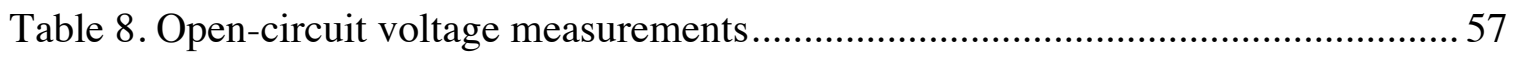




\section{LIST OF FIGURES}

Figure

Figure 1. Diagram of a galvanic cell ................................................................. 7

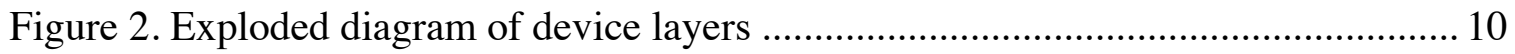

Figure 3. Microfluidic path of activating solvent ................................................... 11

Figure 4. Chemical species evolution of conductive ink .......................................... 14

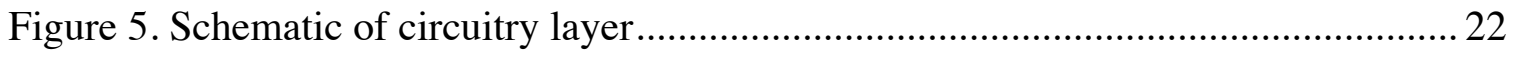

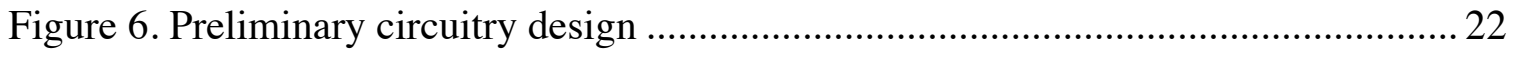

Figure 7. Exploded view of device with printed-circuitry ...................................... 22

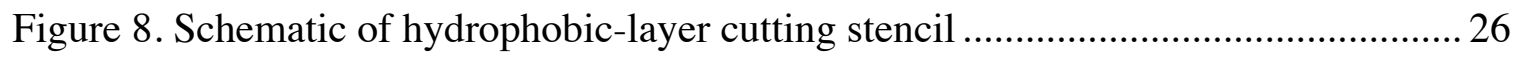

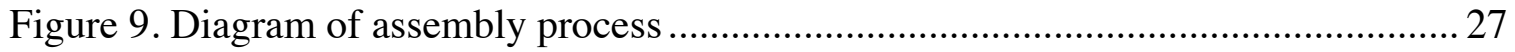

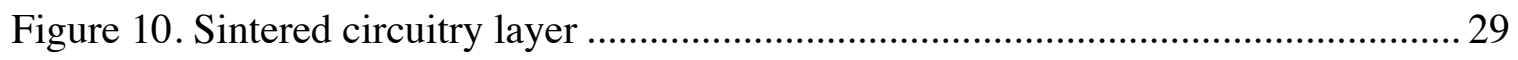

Figure 11. Resistivity of circuitry traces with respect to fold angle .............................. 30

Figure 12. Diffraction pattern of unprinted circuitry layer ...................................... 31

Figure 13. Diffraction pattern of sintered circuitry layer......................................... 32

Figure 14. Two-factor matrix of conductivity threshold ........................................... 33

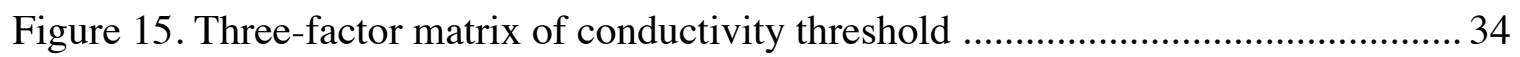

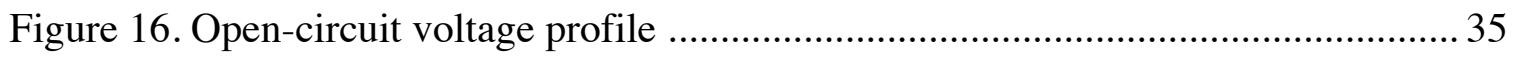

Figure 17. Mean short-circuit current profile ......................................................... 36

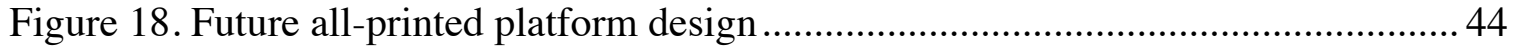




\section{INTRODUCTION}

\subsection{Project Definition}

\subsubsection{Problem Statement}

Microfluidic paper-based analytical devices ( $\mu$ PADs) are ultra-low cost, open-source, scalable, portable, and combustible solutions to the need for biological and chemical sensing assays in resource-limited environments. These devices print hydrophobic channels on paper substrate sheets using solid-wax ink and capitalize on capillary flow to move small-volume liquid samples toward regions with pre-deposited reagents, providing a chemical sensing platform tunable to the analyte. In their current form, they are limited in their inclusion of active assays, powered electronics and automatable features. The microfluidic battery is a multi-layer $\mu \mathrm{PAD}$ with pre-deposited electrolytes and metal-film electrodes, generating electrochemical power from the galvanic potential. As such, active assays and powered electronics have been added to the envelope of solutions able to be addressed by $\mu$ PADs. However, current microfluidic battery designs use copper tape as the circuitry material to deliver terminal voltages spatially with the device layers, limiting automation capability and feature size. Therefore, the integration of printable external circuitry onto the cellulose substrate and into the battery design is valuable for advancing the automation of this chemical sensing platform. The subsequent question to ask is: Can a printable circuitry material be integrated into a microfluidic battery to displace the existing technology used? 


\subsubsection{Central Hypothesis}

A printed circuitry material can be integrated into microfluidic battery design without compromise to battery performance, mechanical properties, packaging, and assembly processes to create an all-printed platform.

\subsubsection{Scope}

The key deliverable was a working prototype of a microfluidic battery with circuitry external to galvanic cells, yet integrated with the substrate, assembly, and function of the device. The scope of this work includes only the material selection, integration, and characterization of the circuitry material. Success was measured by the satisfaction of quantitative, singular, verifiable, non-conflicting, unambiguous requirements set forth in the design section (Defense Acquisition University Press, 2001). The short-circuit current and open-circuit voltage were recorded to define the performance of the battery. The conduction threshold of ink concentration was mapped using a matrix of sintering parameters; the presence of bulk silver was confirmed using x-ray diffraction. To characterize the circuitry's stability to mechanical fatigue, folding was induced and electrical resistance was recorded.

\subsubsection{Limitations \& Constraints}

Limitations of scope narrow the context of this research's findings, but the objective is only to obtain a modicum of knowledge or progress toward the envelope of solutions $\mu$ PADs offer. Thus, the following constraints have been applied to the scope of this investigation: 
- The design is constrained to an existing microfluidic battery platform (Thom, Lewis, DiTucci, \& Phillips, 2013; Thom, Yeung, Pillion, \& Phillips, 2012).

- Chemical sensing functions and features are excluded from the design of the platform.

- The findings of device and ink performance do not extend beyond the context of the cellulose-paper substrate.

- The theoretical value of percolation threshold is not defined, nor is the error between it and the empirically established value quantified.

\subsection{System Requirements}

The system requirements set forth can be divided into three tiers of relevance: the circuitry material, the integration of that material into the device and the platform interface. The circuitry material must be conductive such that the performance of the microfluidic battery is not inhibited. The circuitry material must also be stable on the substrate over time to allow for assembly and packaging. The integration of the printed circuitry must be mechanically robust and low-temperature to preserve the flexibility of the paper substrate. As an all-printed platform, the device must enable data collection sufficiently long enough for existing assays or sensing. The platform must also be able to interface with the electronic components used in such assays. 


\section{BACKGROUND}

This master's thesis integrates three key technology platforms to test the central hypothesis: wax printing of microfluidic paper-based analytical devices ( $\mu$ PADs), microfluidic batteries and reactive silver-nanoparticle conductive ink.

\subsection{Wax Printing}

Wax printing is the micro-patterning of solid wax as ink on sheets of cellulose-paper substrate to create simple, inexpensive microfluidic devices. These wax traces are patterned on paper using various printing methods such as photolithography, screenprinting, and solid-wax inkjet printers. By heating under prescribed profiles, the solid wax wicks through the depth/thickness of the paper, creating a sealed channel for microfluidic flow between hydrophobic traces (Carrilho, Martinez, \& Whitesides, 2009). Microfluidic paper-based analytical devices ( $\mu$ PADs) are chemical or biological assays that capitalize on both the nano-scale phenomena of microfluidics and inexpensive, simple fabrication of wax printing to create diagnostic tests. Hydrophobic channels are patterned onto cellulose paper substrates that direct flow of liquid samples to detection pads. Reagents required for chemical sensing of the analyte are pre-deposited onto the cellulose substrate at specific locations and concentrations. Qualitative results (e.g. color change) can be designed into the chemical sensing reaction; quantitative data collection uses external equipment (e.g. glucose readers, spectrophotometers, cell phones) to interpret the assay (Carrilho et al., 2009; Cate, Adkins, Mettakoonpitak, \& Henry, 2015; Park, Li, McCracken, \& Yoon, 2013). 
These innovations are particularly well suited for remote testing in resource-limited countries because they are built on an ultra-low-cost platform and carry minimal effort for end-of life handling (i.e. disposal via burning). The minimal embedded energy and safe, reliable disposal circumvents the embedded high infrastructure costs of typical laboratory testing (Carrilho et al., 2009; Cate et al., 2015) .

The salient lesson is that no single $\mu \mathrm{PAD}$ technology has proven superior. The power and versatility of $\mu \mathrm{PADs}$ is their tunable nature: devices can vary in substrate, patterning method, reagent composition, target analyte, and data collection. The number of permutations is countless, as are the possible applications.

\subsection{Microfluidic Battery}

Microfluidic batteries are "galvanic cells incorporated directly into a multilayer paperbased microfluidic device." Aqueous solutions of electrolytes for each electrode half-cell and the salt bridge that connects the half-cells are pre-deposited onto different layers of the device; the water is evaporated before assembly. The deposited electrolytes contact the metal foil electrodes and are connected via a salt bridge, creating a full galvanic cell. Upon the addition of a solvent (in this case, deionized water), the ions of the electrolyte and salt are remobilized in solution, and the reduction and oxidation reactions at the electrodes can proceed. Thus, these devices are "activated" or "turned on" via the addition of a fluid. Furthermore, the use of microfluidic channels to connect each halfcell adds to the relevance of the battery's "fluidic" nature. Determining factors of the power generated are volumetric density of ions, feature size, arrangement of cells, and 
choice of electrode metals. The influence of each is well characterized and tunable for desired performance (Thom, Lewis, DiTucci, \& Phillips, 2013).

To create a microfluidic battery, a multi-layer device using alternating layers of hydrophobic dual-sided adhesive layers and hydrophilic paper layers with per-patterned wax-printed hydrophobic channels is assembled to direct deionized water to an unsaturated $\mathrm{NaNO}_{3}$ salt bridge connecting two metal electrodes, specifically aluminum and silver (Thom, Lewis, DiTucci, et al., 2013; Thom et al., 2012). The approach of this study is based on this design, but deviates from prescribed electrolyte densities as well as hydrophobic and circuitry materials.

\subsubsection{Galvanic Potential}

The generation of electrical potential using galvanic cells is well established and the core principle of the microfluidic battery. An electrical circuit of electron and ion channels is connected to two dissimilar metals, creating a complementary flux of dissociated electrons and ions from the corresponding reduction-oxidation reaction at each electrode surface. (Figure 1, [Hazmat2, 2012]) The resulting flow of electrons through the external circuitry is used to power an electrical load until the reaction becomes thermodynamically stable and nonspontaneous. Upon reaching this equilibrium, charges no longer conduct and current through the load diminishes and reaches a terminus. 


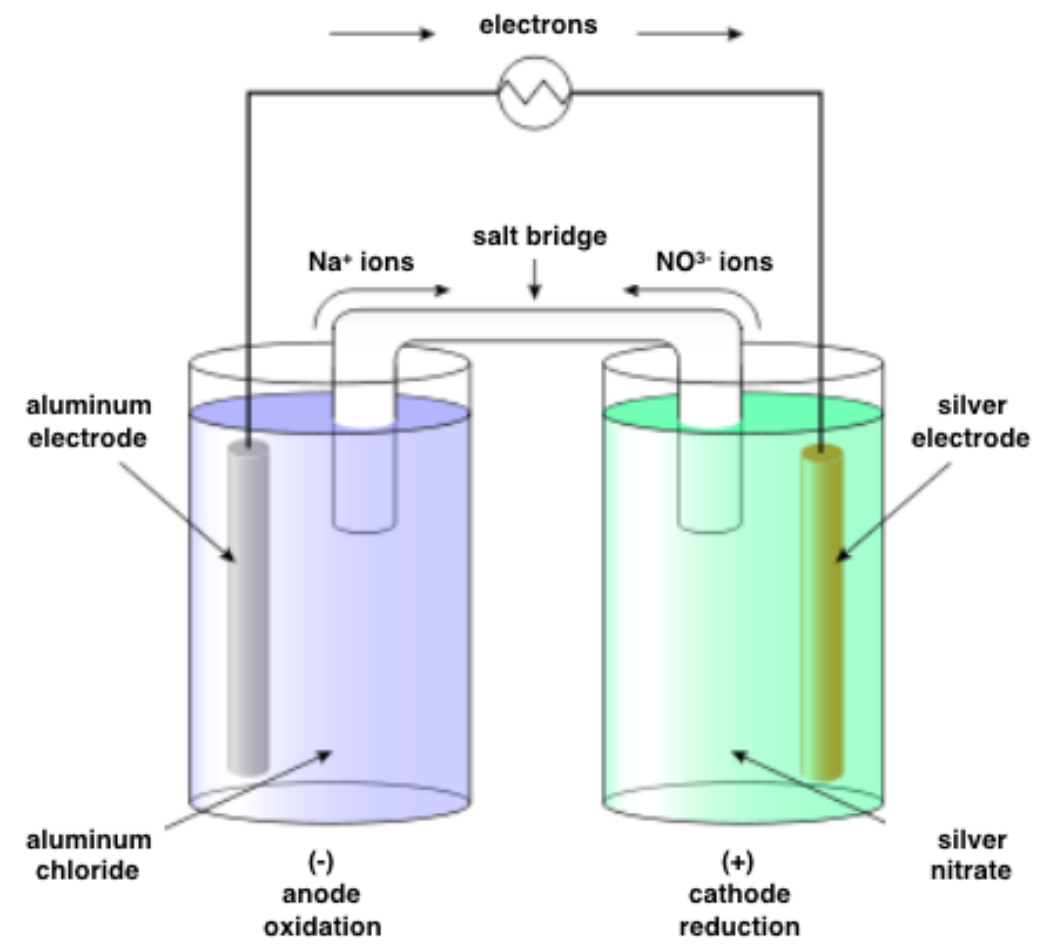

Figure 1. Diagram of a galvanic cell

Given the half-cell reactions,

$$
\begin{aligned}
& A g^{+}+e^{-} \rightleftharpoons A g(s) \\
& A l^{3+}+e 3^{-} \rightleftharpoons A l(s)
\end{aligned}
$$

and their associated standard electrode potentials,

$$
\begin{aligned}
E_{A g}{ }^{\circ} & =0.7996 \mathrm{~V} \\
E_{A l}{ }^{\circ} & =-1.662 \mathrm{~V}
\end{aligned}
$$

we expect a standard electrode potential $\left(E_{\text {cell }}^{\circ}\right)$ of the redox reaction

$$
E_{\text {cell }}^{\circ}=E_{\text {cathode }}^{\circ}-E_{\text {anode }}^{\circ}
$$


to yield a theoretical potential of $2.46 \mathrm{~V}$. This value agrees with our expectations of spontaneity $\left(\Delta G^{\circ} \leq 0\right)$ described by the standard electrode potential identity of Gibb's free energy of as

$$
\Delta G^{\circ}=-n F E_{\text {cell }}^{\circ}
$$

where $n$ is the molar ratio of electrons to reaction products and $F$ is the Faraday constant of $9.64850332\left(10^{4}\right) \mathrm{C} \mathrm{mol}^{-1}$. Furthermore, this theoretical potential value is $35 \%$ above the design requirement for threshold voltage discussed in later sections, so the silveraluminum system is appropriate for use in this device. 


\subsubsection{Layer Functions}

There are eight (8) discrete layers of alternating hydrophobicity of the microfluidic battery, each serving a separate function required for power generation. (Table 1)

Table 1. Individual layer functions

\begin{tabular}{|c|c|c|c|}
\hline Layer & Substrate & Patterning Method & Function \\
\hline 1 & Adhesive tape & Mechanical stamping & Sample input \\
\hline 2 & Cellulose paper & Solid-wax inkjet & Distribute sample \\
\hline 3 & Adhesive tape & Mechanical stamping & Salt bridge input \\
\hline 4 & Cellulose paper & $\begin{array}{c}\text { Solid-wax inkjet; } \\
\mathrm{NaNO}_{3} \text { pre-deposited }\end{array}$ & Reconstitute salt bridge \\
\hline 5 & Adhesive tape & Mechanical stamping & Isolate electrode pads \\
\hline 6 & Cellulose paper & $\begin{array}{c}\text { Solid-wax inkjet; } \\
\mathrm{AgNO}_{3} / \mathrm{AlCl}_{3} \text { pre-deposited }\end{array}$ & $\begin{array}{l}\text { Electrolyte conduction; } \\
\text { redox interface }\end{array}$ \\
\hline 7 & Adhesive tape & Mechanical stamping & Affix electrodes \\
\hline 8 & Copper tape & Mechanical cutting & External circuitry \\
\hline
\end{tabular}


By constricting the exterior dimensions of each layer to be identical, wax-printed features and vertical channels remain aligned during assembly. As an added benefit, alignment errors after assembly can be identified visually. (Figure 2)

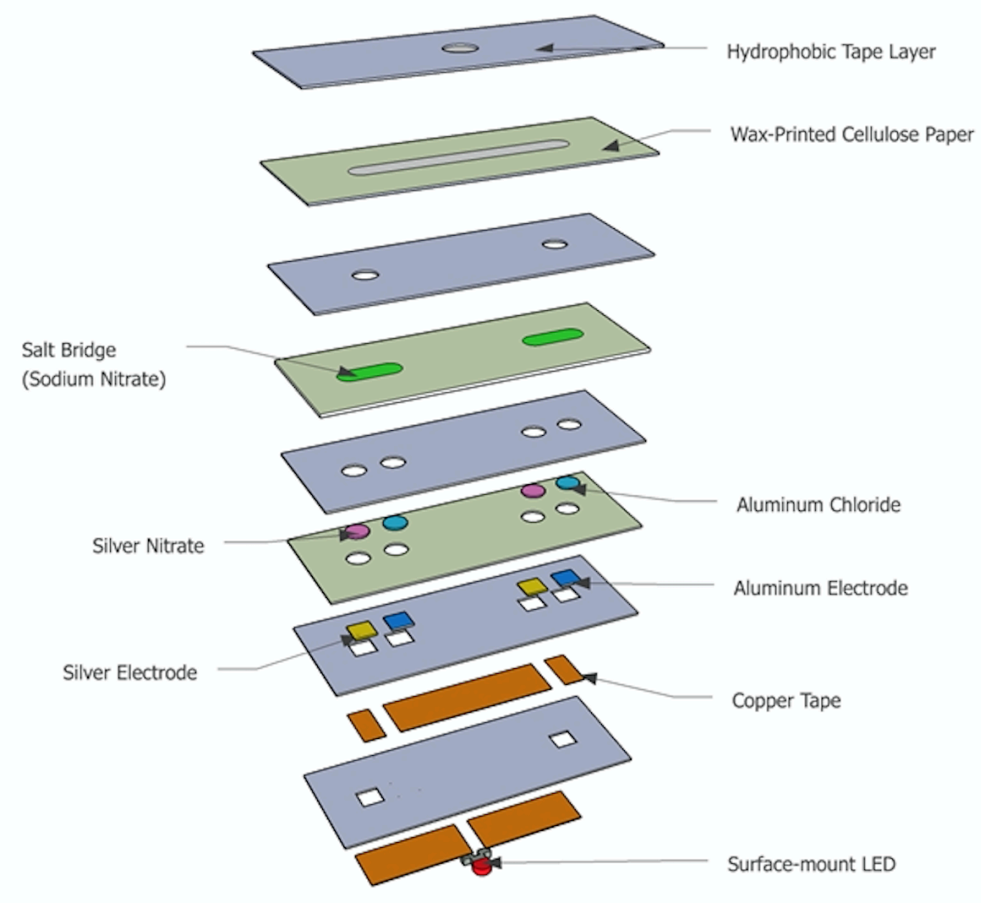

Figure 2. Exploded diagram of device layers

The microfluidic battery not only capitalizes on the capillary flow of deionized water input; the water itself reconstitutes the pre-deposited salt bridge and electrolytes by dissolving these reagents back into solution. The ion path of the galvanic cell is thus activated upon the reconstitution of this salt bridge. (Figure 3) The electron path provided by copper-tape circuitry is already attached to the opposing face of the electrodes, ready to conduct upon "turn-on" of the device. 


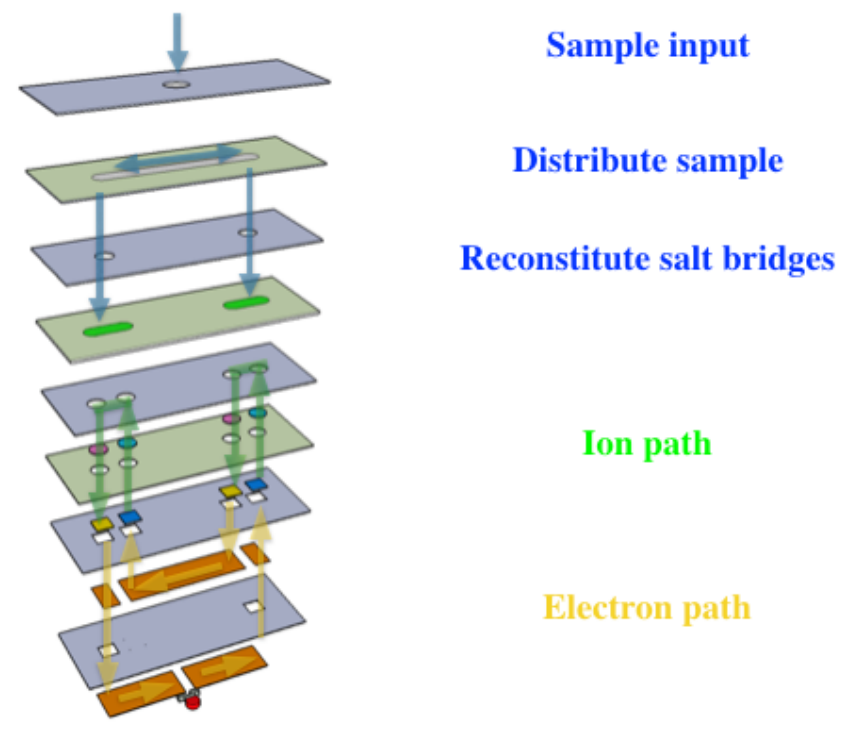

Figure 3. Microfluidic path of activating solvent

\subsection{Conductive Ink}

Pioneers of the industry have attempted mapping and addressing the needs for circuitry in devices that use electrical potential for $\mu \mathrm{PAD}$ functions. In the interim, copper tape has been nearly ubiquitous placeholder for prototyping.

A thorough investigation of solid metal wires using zinc or tin, attached to the substrate with a bead of conductive epoxy, has been conducted to mimic techniques used currently in the printed-circuit board industry (Siegel et al., 2010). Patterning methods are well established and abundant for metallic wires, such as chemical or physical deposition, and have already been implemented on a variety of substrate materials. Such methods lend themselves to high accuracy and small feature size, both of which are particularly well suited for automation. However, high equipment, process and material costs prohibit their viability for salable platforms. 
Graphite electrodes and circuitry have drawn significant interest for the inexpensive, numerous carbon precursors, variety of solution chemistries, and tunable electrical properties of different carbon allotropes. Inkjet printing methods of carbon-based inks are numerous, readily available and inexpensive, but uniformity and reliability of conductive traces is limited by the capacitive properties of graphite. Higher conductivity allotropes such as carbon nanotubes can be implemented but increase cost considerably and their end-of-life disposal is poorly understood (Lan et al., 2013).

Conductive nanoparticle inks exhibit attractive qualities for flexible printed media. Several permutations of investigations varying patterning method and copper, silver, or gold nanoparticles have been conducted, capitalizing on elevated homologous temperatures and conductivity limits. However, these precursors costs and processing temperatures can be prohibitive and thus limit their applicability. Copper and gold nanoparticles are already used in biomedical sensing, but copper oxide formation is difficult to mitigate without complex sintering chemistries. Additionally, gold is a conflict mineral and contradicts the purpose of servicing resource-limited or contaminated environments (Adkins, Boehle, \& Henry, 2015; Cate et al., 2015; Shari, Ghobadian, Cavalcanti, \& Hashemi, 2015; Thom, Lewis, DiTucci, et al., 2013).

Conductive inks are electrically conductive liquid solutions that are stable under ambient conditions but, upon printing, their solvent evaporates leaving solid particulates adhered to the substrate media. Conductive inks allow for the deposition of conductive material with high precision and resolution using conventional patterning methods such as screen and inkjet printing. These materials are especially relevant when patterning on substrates such as paper or PET which require low processing temperatures (under $200^{\circ} \mathrm{C}$ ) (Glavan, 
Walker, Lewis, Whitesides, \& Link, 2016; Kim \& Moon, 2005; Perelaer, Abbel, Wünscher, Jani, \& Lammeren, 2012). Ink-based circuitry complements both the ultralow-cost basis and manufacture scaling of $\mu$ PADs. Their incorporation is especially simpler when patterning techniques such as screen or inkjet printing are utilized because both are established methods and can be automated.

This study implements silver-nanoparticle conductive ink that is vetted specifically for high conductivity, proven printability, and minimal oxide formation. In addition, this conductive ink is well suited for the mild temperatures required for devices using the cellulose paper substrate as a platform. Furthermore, the incorporation of the conductive ink can be seamless with the multi-layer design of microfluidic batteries.

\subsubsection{Synthesis}

The conductive ink is synthesized through a modified Tollens' reaction as prescribed in (Walker \& Lewis, 2012). The resulting solution is a diamminesilver (I) complex in an ammonium formate solvent containing $22 \mathrm{wt} \%$ silver. Stable in ambient conditions up to six (6) months, the solution only precipitates silver particles when exposed to air (i.e. when patterned on substrate). Upon printing, the labile amine ligands evaporate and silver nanoparticles ranging from 2-200 $\mathrm{nm}$ in diameter remain. (Figure 4) By design, sintering

at temperatures below $100^{\circ} \mathrm{C}$ are purported to achieve the conductivity of bulk silver within 15 minutes (Walker \& Lewis, 2012). 


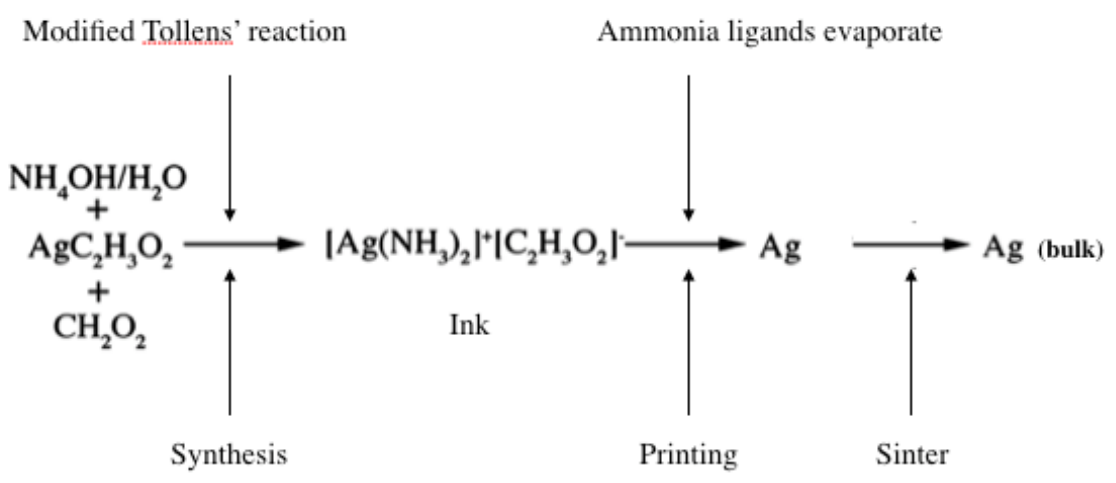

Figure 4. Chemical species evolution of conductive ink

\subsubsection{Percolation Theory}

Percolation theory in the context of material science is the mapping of bonding between sites of a given three-dimensional network. Within any such network there exists a threshold, $p_{c}$ above which the sites are continuously connected within a given volume. The percolation threshold, $p_{c}$, is defined as the "concentration (occupation probability) $p$ at which an infinite cluster appears for the first time in an infinite lattice" (Christensen, 2002). In the context of sintering solid metal particles, it can be used to define the minimum concentration of dispersed particles required for atomic diffusion. Above this critical value, conductive networks have sufficient bonding for electrical conductivity and atomic diffusion to occur (George \& Sebastian, 2008). In the case of this study, is has been shown that "conductivity in conductor-insulator system is zero below $p_{c}$ and increases with a power law above $p_{c}$ " (Heitjans \& Kärger, 2005). Theoretical values of this threshold can be determined using simulation and modeling, given various particle geometry, size distribution, and concentration, but empirical approaches employing percolation models have been shown to be highly accurate for metallic silver networks of 
submicron particle size (Deptuck, Harrison, \& Zawadzki, 1985; Holm \& Cima, 1989). In this study, the threshold will be mapped operationally as a minimum ink concentration, circumventing the need for simulation or modeling.

\subsubsection{Conductivity}

Conductivity, the reciprocal of resistivity, is defined (for elemental metallic solids) as a material's ability to conduct electrical current, often expressed as

$$
\sigma=n_{e} q \mu_{e}
$$

where $n_{e}$ is the number density per volume of conduction electrons available, $q$ is the elementary charge of an electron $(\mathrm{C})$, and $\mu_{\mathrm{e}}$ is the electron mobility $\left(\mathrm{m}^{2} \mathrm{~V}^{-1} \mathrm{~s}^{-1}\right)$. The density and charge of electrons cannot be changed; therefore, the only method accessible to increase conductivity is the increase of electron mobility by manipulating the material's crystal lattice. The determining factor of electron mobility is scattering time, even more so in metallic nanoparticles (as is the case for this research), where dimensional quantum confinement and interfacial/boundary scattering are nontrivial. Reducing the short-range disorder causing interfacial scattering, namely surface roughness, results in a disproportionate/nonlinear increase in electron mobility (Ferry, Goodnick, \& Bird, 2009). It has been shown that for networks of silver nanoparticles with high surface area to volume ratio, the interfacial resistance is appreciable, which attributes to the deviation of conductivity from their bulk state. Densification increases the number of "percolating pathways for electron" travel and lowers interfacial resistance, resulting in an increase in conductivity (or decrease in resistivity) (Perelaer et al., 2012). 
The resistance $(\Omega)$ of bulk materials, modeled as a simple discrete wire, is described by

$$
R=\rho \frac{L}{A}
$$

where $\rho$ is resistivity $(\Omega \mathrm{m}), A$ is cross sectional area $(\mathrm{m})$ of the electron path, and $L$ is the length $(\mathrm{m})$ of wire. As densification increases and additional electron pathways are established, the effective area of conduction also increases. Therefore, the integral consideration for conductivity of silver nanoparticle inks is the degree to which the network is connected and sintered.

\subsubsection{Sintering}

Sintering is the process of treating solid, discretely separate particulates with temperature and/or pressure to create a continuous network of grains from a powder precursor. The applied kinetic energy creates a diffusion gradient between the interior and exterior of the network. The surface and grain-boundary diffusion of atoms fuse the interfaces of these particles together, after which sufficient diffusion pathways are established for lattice diffusion. Upon the change in atomic transport regime, exterior atoms move inward along the gradient and occupy the cavities/voids in the powder network, resulting in a reduction in volume (i.e. densification) and grain growth. This process is differentiated from other heat treatments by the absence of liquefaction, as the treatment temperatures are significantly below the material melting point. The temperature at which a material is sintered is expressed as the homologous temperatures, defined as the fraction of the material's melting temperatures $\left(\mathrm{T} / \mathrm{T}_{\mathrm{m}}\right)$. This homologous temperature is a key thermodynamic variable for modulating which regime/mechanism of atomic diffusion (i.e. surface, grain boundary, or lattice) is dominant in a crystal lattice. It is then deduced 
that melting temperature is a defining material property in the sinter profile of a precursor (Knoerr, Kraft, \& Schletz, 2010; Rahaman, 2003).

It has been established that particles with especially high surface area to volume ratios experience a decrease in melting temperature (Asoro, Damiano, \& Ferreira, 2009; Greer $\&$ Street, 2007). The change in surface energy's contribution to free energy of these systems account for the non-linear deviation from the calculated relationship between melting point and particle size, namely below $100 \mathrm{~nm}$ (Allen, Bayles, Gile, \& Jesser, 1986). These nano-scale phenomena and size effects are most applicable to solid particles with spherical geometry (Wronski, 2002). In the case of nanometer-sized particles, a decrease in melting temperature corresponds directly to increases homologous temperature and therefore improves the sintering efficiency at lower temperatures. 


\section{DESIGN}

\subsection{Functional Requirements}

This study approaches the hypothesis by fabricating a prototype device that adds a conductive-ink circuitry layer to an existing microfluidic battery design derived from Thom et al.

The functional requirements are prescribed to be quantitative, singular, verifiable, nonconflicting and unambiguous statements, translated from the system requirements and tested in methods conforming to standard practices for quantification (Cate et al., 2015; Defense Acquisition University Press, 2001; Shari et al., 2015):

- Absolute resistance accounting for no more than 5\% loss of power generated.

- Circuitry material must be chemically stable at $25^{\circ} \mathrm{C}$ in air on the cellulose substrate for 30 days (Glavan et al., 2016).

- Device must be assembled at temperatures below $200^{\circ} \mathrm{C}$ to preserve mechanical properties of the substrate.

- Functionality at bend angle $(\theta)$ of $90^{\circ}$ must be preserved with maximum $20 \%$ loss of conductivity at $180^{\circ}$ (Siegel et al., 2010).

- Battery must generate power for a full-duration half-max minimum of 5 minutes (Ellerbee et al., 2009; Park et al., 2013).

- Battery must maintain power levels above the voltage threshold and forward current of commercially-available LEDs, specifically 1.8V and $100 \mu \mathrm{A}$ (LITEON Optoelectronics, 2006). 


\subsection{Microfluidic Battery}

\subsubsection{Design Adaptations}

The design of the fluidic battery was derived from the work conducted by Thom et al. These batteries have proven effective in generating sufficient power for on-chip components (including LEDs). This solution employs design permutations adapted from the original research and chosen as a compromise between feature size, complexity, and maximum potential (Thom, Lewis, DiTucci, et al., 2013; Thom et al., 2012). These compromises include:

- A linear arrangement of cells in series was chosen because it raises operating voltage $(2.7 \mathrm{~V})$ to above the threshold of common surface-mount LEDs $(1.8 \mathrm{~V})$.

- A $9 \mathrm{~mm}^{2}$ electrode size extends the device "on" time to minutes, which is experimentally favorable for testing and characterization. Additionally, this electrode size is a footprint proportionate to the size of interfacing features, namely the salt bridge. Alignment is paramount during assembly; larger features can be more forgiving of human error.

- A choice of metal-salt electrolyte density of $400 \mu \mathrm{g} \cdot \mathrm{mm}^{-3}$ of $\mathrm{AgNO}_{3}\left(\right.$ or $\left.\mathrm{AlCl}_{3}\right)$ was chosen because although it has the highest rate of current decay, it increases peak current that, for the sake of prototyping will be easier to characterize.

- Choice of salt bridge electrolyte and concentration was prescribed as 9.6 $\mu \mathrm{mol} \cdot \mathrm{mm}^{-3} \mathrm{NaNO}_{3}$ and requires no tailoring for performance. The maximum salt bridge volume $\left(\sim 2.07 \mathrm{~mm}^{3}\right)$ was chosen despite the increase in resistance, delay of turn-on time and decrease in maximum current. These design sacrifices were made for the decrease in current decay rate and increase in assembly ergonomics. 
Given the balance between the choices of electrolyte density and salt bridge volume a moderate-duration and high-power current profile is established.

\subsubsection{Hydrophobic Layer Material}

To circumvent the difficult handling and processing (i.e. laser cutting) of the carpenter's tape prescribed in the original battery design, the hydrophobic layer material was chosen to be $3 \mathrm{M}^{\mathrm{TM}}$ High Performance Acrylic Adhesive 200MP Transfer Tape. Model 467MP was specified for the following reasons (3M Corporation, 2014):

- Adhesive thickness $0.06 \mathrm{~mm}$

- This feature provides a smaller z-axis travel of the sample within vertical channels of the hydrophobic layers

- Double sided, double backed

- This feature provides superior handling during cutting and assembly stages. Furthermore, contamination is reduced because exposure time is minimal

- Excellent solvent resistance

- The presence of metal-salt electrolytes creates high-activity solutions within the device that interface with the hydrophobic layer

- High shear strength

- Delamination is a critical failure for these devices, thus preservation against slippage and edge lifting is required during assembly, packaging, and distribution stages 
- Water and humidity resistance

- Minimal diffusion of water into the hydrophobic layer is desired

- Long-term stability with moderate temperature range

- Subassemblies that include the tape layer may require sintering in future platform designs and this process-flexibility should not be precluded.

\subsubsection{Printed Circuitry Layer}

The eighth (8th) layer must satisfy each purpose of the copper tape, primarily the connection between terminals of galvanic cells. As a secondary purpose, it should simplify assembly and decrease misalignment error.

The proposed design consists of a rectangular center trace connecting the two galvanic cells, and two side "L"-shaped traces rerouting terminal voltage to a $300 \mu \mathrm{m}$ gap, the minimum allowable by wax printing but maximum for the surface-mount LED footprint. Figure 5) The side trace channel width minimum of $2 \mathrm{~mm}$ is required for wetting of the precursor solution; the $4 \mathrm{~mm}$ base acts as a reservoir during deposition. In previous design iterations, smaller channel widths were too narrow for ink wetting and thus did not receive sufficient solute for sintering. (Figure 6) The center trace is spaced specifically to align a wax trace between the electrodes with each pair. By matching the exterior dimensions to layers 1-7, alignment is unambiguous and less error-prone during assembly. 


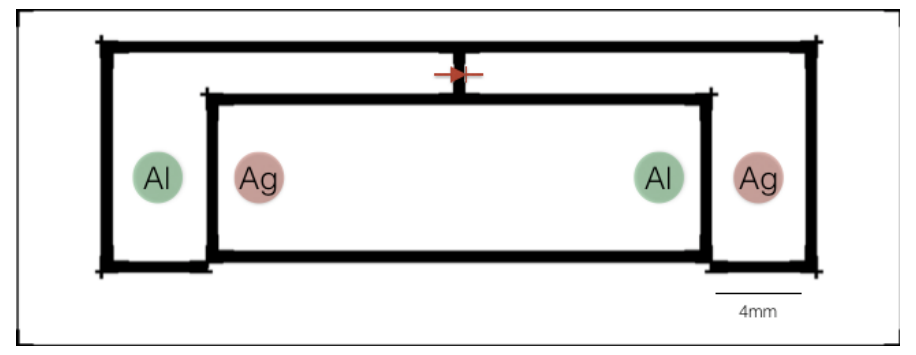

Figure 5. Schematic of circuitry layer

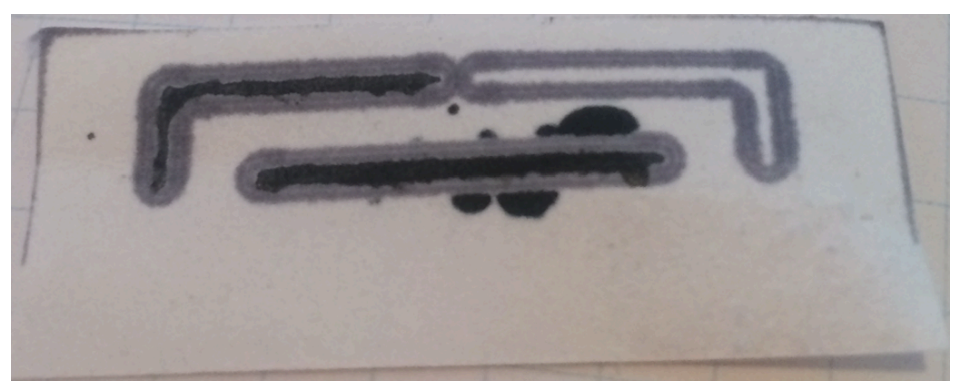

Figure 6. Preliminary circuitry design

The value of incorporating a printed circuitry layer as an all-printed platform is visible when superimposed on the original battery design. (Figure 7)

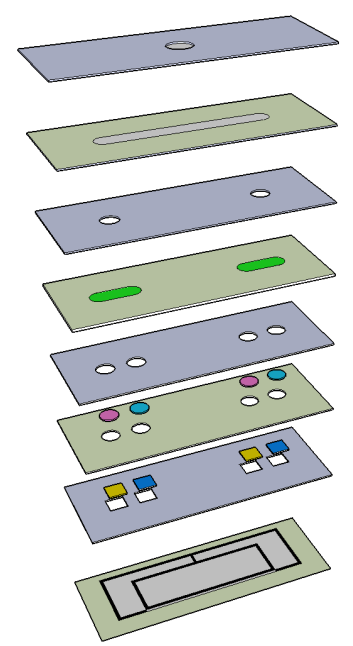

Figure 7. Exploded view of device with printed-circuitry 


\subsection{Conductive Ink}

The selection to use mild-temperature silver-nanoparticle conductive ink can be explained by the relation between its performance and the functional requirements:

- The silver becomes incorporated with substrate pore network after sintering, so fatigue behavior may be unlike the graphite electrodes or zinc metal traces of other researchers' attempts. Additionally, printing is required only once per trace because conductivity is achieved on both sides (Siegel et al., 2010).

- The mild temperature sintering (below $200^{\circ} \mathrm{C}$ ) is attractive for preserving substrate mechanical properties (i.e. flexibility) (Walker \& Lewis, 2012).

- The theoretical conductivity within reach is sufficient for preserving the power generated (Walker \& Lewis, 2012).

- The ink has proven scalable for automated printing methods (Walker \& Lewis, 2012).

- Low-viscosity of the solution means deposited liquid will conform to the hydrophobic barriers of wax-printed design, so creating complex circuitry traces will be nested in the most robust process of assembly, namely the wax printing.

- The ink is stable for 3 months so the burden of storage is low and it experiences no appreciable loss in potency.

\subsubsection{Sinter Profile}

The formation of a "well-connected, continuous, and conductive network" has been shown to develop sharply at sintering temperatures above $150^{\circ} \mathrm{C}$ on silicon substrates. The reliability of this network formation increases with peak temperature, yet isothermal 
holds are sufficient to activate sintering provided they are above the critical value (Greer $\&$ Street, 2007). It is deduced that densification increases significantly above this temperature, because, given a large increase in homologous temperature when above the percolation threshold, a change is expected in the dominant diffusion mechanism (namely, surface diffusion initiates fusion and creates necks, then switching to grain boundary diffusion which removes void, densifies, and grows grains) (Rahaman, 2003). Therefore, the test matrix of sinter profiles is simplified by using isothermal holds marginally below, at, and above $150^{\circ} \mathrm{C}$. 


\section{EXPERIMENTAL METHOD}

\subsection{Printed Features}

The solid wax ink was patterned onto Whatman Chromatography No. 1 paper using a Xerox Phaser $6500 \mathrm{~N}$ inkjet printer. Sheets of patterned paper were heated in an MTI Compact Forced Air Convection Oven for $190^{\circ} \mathrm{C}$ for 120 seconds to cure hydrophobic channels. After individual layers were cut, $4 \mu \mathrm{L}$ of saturated $\mathrm{NaNO}_{3}$ solution was deposited using a micropipette onto the salt bridge channel and left in air for 30 minutes to evaporate. Separately, $3 \mu \mathrm{L}$ volumes of $1 \mathrm{M} \mathrm{AlCl}_{3}$ and $3 \mathrm{M} \mathrm{AgNO}_{3}$ solutions were deposited via micropipette into their respective electrolyte pads and evaporated.

\subsection{Cutting/Stamping}

Adhesive layers are stamped and punched using a cutting stencil that aligns "vertical channels", or holes, to their correct position relative to the exterior dimensions of the device (Figure 8) With this stencil, one copy of each hydrophobic layer is cut and thus wastes minimal material on duplicates. Alignment during assembly is determined at the exterior corners for each layer, a user-friendly feature implemented after fabricating exploratory stencil prototypes. 


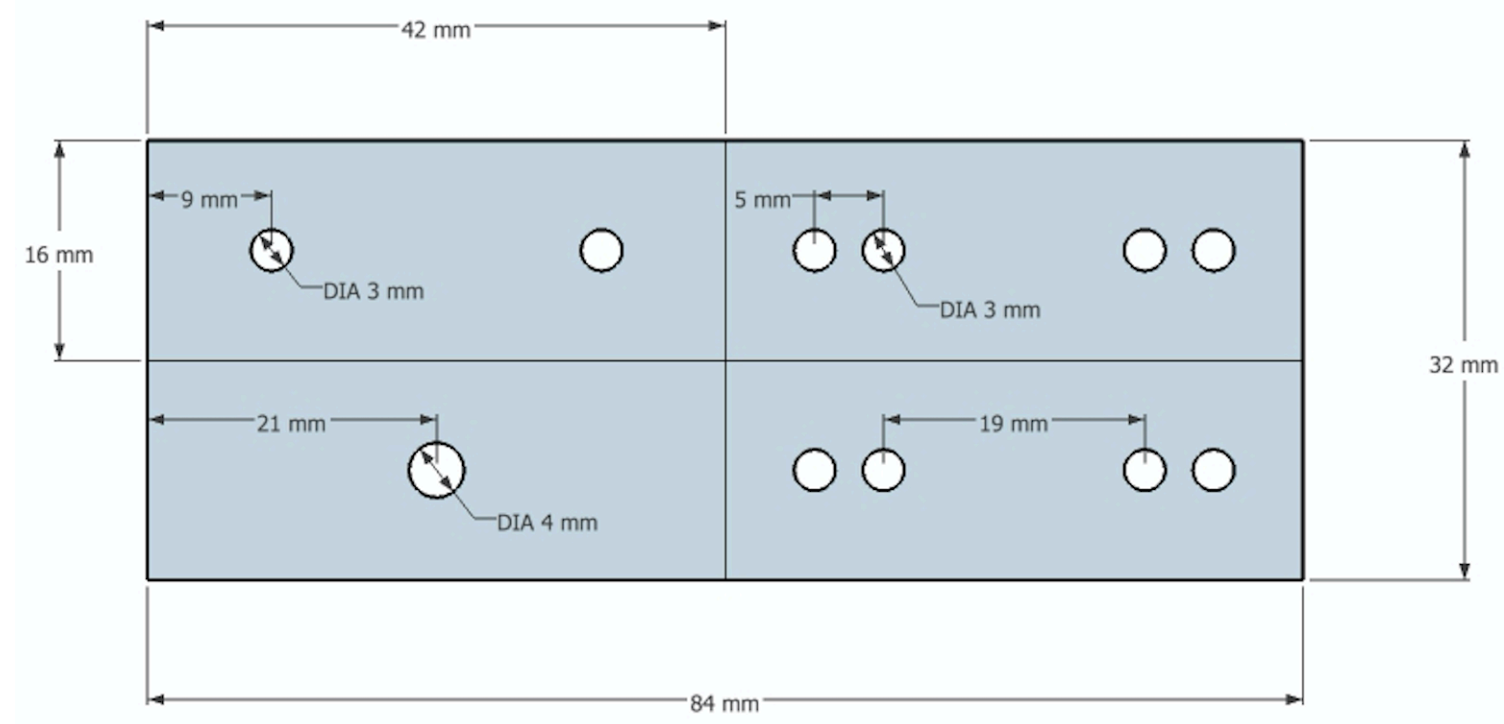

Figure 8. Schematic of hydrophobic-layer cutting stencil

\subsection{Printed Circuitry}

Multiple sheets of equally sized $(22 \mathrm{~mm}$ x $11 \mathrm{~mm})$ wax-printed channels on paper substrate received conductive ink printing via micropipette and varied in sintering profile. The sinter profile prescribed by literature of $90^{\circ} \mathrm{C}$ at 15 minutes failed in preliminary testing, most likely from a change in substrate. Research of a comparable ink found that temperatures above $130^{\circ} \mathrm{C}$ exhibit reliable sintering on similar media. Additionally, it was found that higher-temperature isothermal-holds performed equally to multi-stage, lower-temperature sinter profiles (Greer \& Street, 2007). Thus, an isothermal hold of $150^{\circ} \mathrm{C}$ was chosen and samples were varied in treatment times from 15 to 90 minutes (in non-linear increments) and from ink concentrations of $10-100 \mu \mathrm{L} \cdot \mathrm{mm}^{-3}$ in $(10 \mu \mathrm{L}$ increments). Upon identifying a narrower range of ink concentrations and sinter times exhibiting significant changes in resistivity, a three-factor experiment (varying in sinter 
time, sinter temperature, and ink concentration) was performed to highlight the ink's conductivity threshold. After print parameters of sufficient performance were identified, circuitry traces (layer 8) were printed, sintered, and then included in assembly.

\subsection{Fabrication}

Three processes, namely the electrolyte deposition, preparation of printed circuitry, and mechanical stamping of tape layers, are conducted in parallel to prepare componentry for the assembly stage. (Figure 9) During assembly, $2 \mathrm{~mm}$ diameter discs of TechniCloth TX609 cellulose/polyester unaligned fiber wipes are cut and inserted in the vacancy of hydrophobic tape layers to reduce the empty space. These discs saturate and swell to create a conduit for capillary flow between layers, reducing the minimum sample volume required. Fully assembled devices are compressed using a $35 \mathrm{lb}$ weight for 30 minutes.

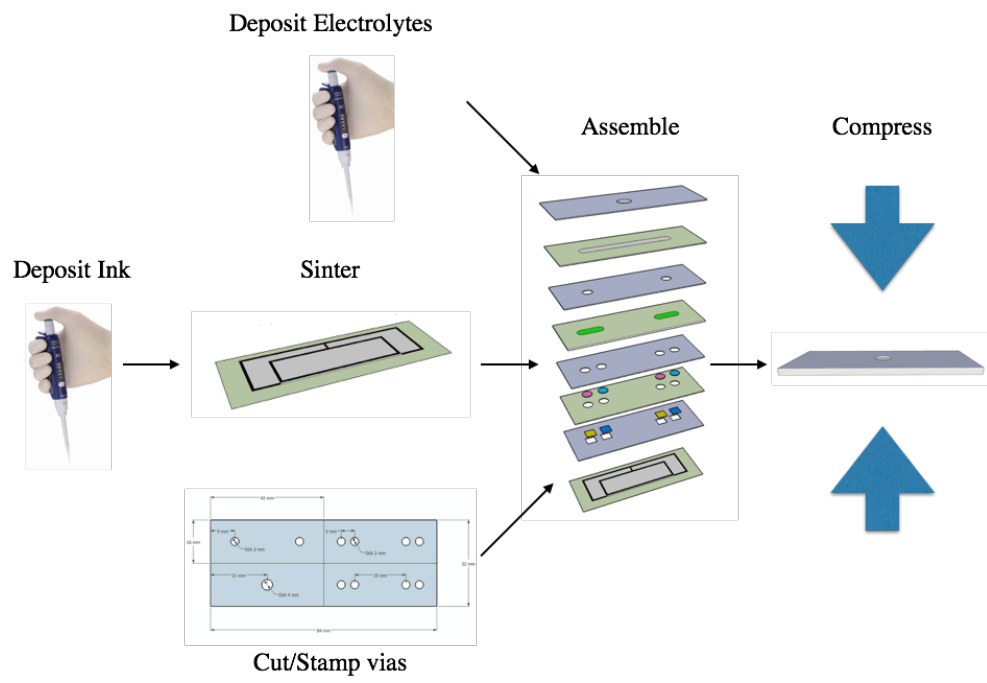

Figure 9. Diagram of assembly process 


\section{RESULTS}

Several characterization methods were implemented to quantify the device's performance in terms of the requirement metrics. The resistance of sintered circuitry traces measured the absolute power loss through the traces as well as their stability over time. The change in resistance of printed traces upon folding at angles varying from $-180^{\circ}$ (acute folding) to $180^{\circ}$ (obtuse folding) measured flexibility. X-ray diffraction confirmed the presence of bulk silver upon sintering. Resistance value measured after samples exposed to a matrix of sinter times, sinter temperatures, and ink concentrations mapped the conductivity threshold. Open-circuit voltage and short-circuit current profiled the power generation of the battery with the print-optimized circuitry included.

\subsection{Resistivity}

Resistance of the center trace and side traces was measured using an Innova 3320 digital multi-meter and observed to be $0.7 \pm 0.2 \Omega$ and $1.1 \pm 0.4 \Omega$, respectively. (Figure 10) These values remained stable in ambient conditions for 30 days without noticeable change in resistance. Compared to the internal resistance of the battery (over $2.53 \mathrm{k} \Omega$ ) these values account for a less than $0.1 \%$ loss in battery power. Trace thickness was measured using Vernier calipers and found to vary significantly, from as low as the cellulose paper's thickness $(0.18 \mathrm{~mm})$ to as large as $0.5 \mathrm{~mm}$. A resistivity range of 75 to $21110^{-5} \Omega \cdot \mathrm{m}$ was calculated, multiple orders of magnitude from the theoretical value of bulk silver 1.6 to $2 \times 10^{-8} \Omega \cdot \mathrm{m}$ at $300 \mathrm{~K}$ (Greer \& Street, 2007; Tanner \& Larson, 1968). 


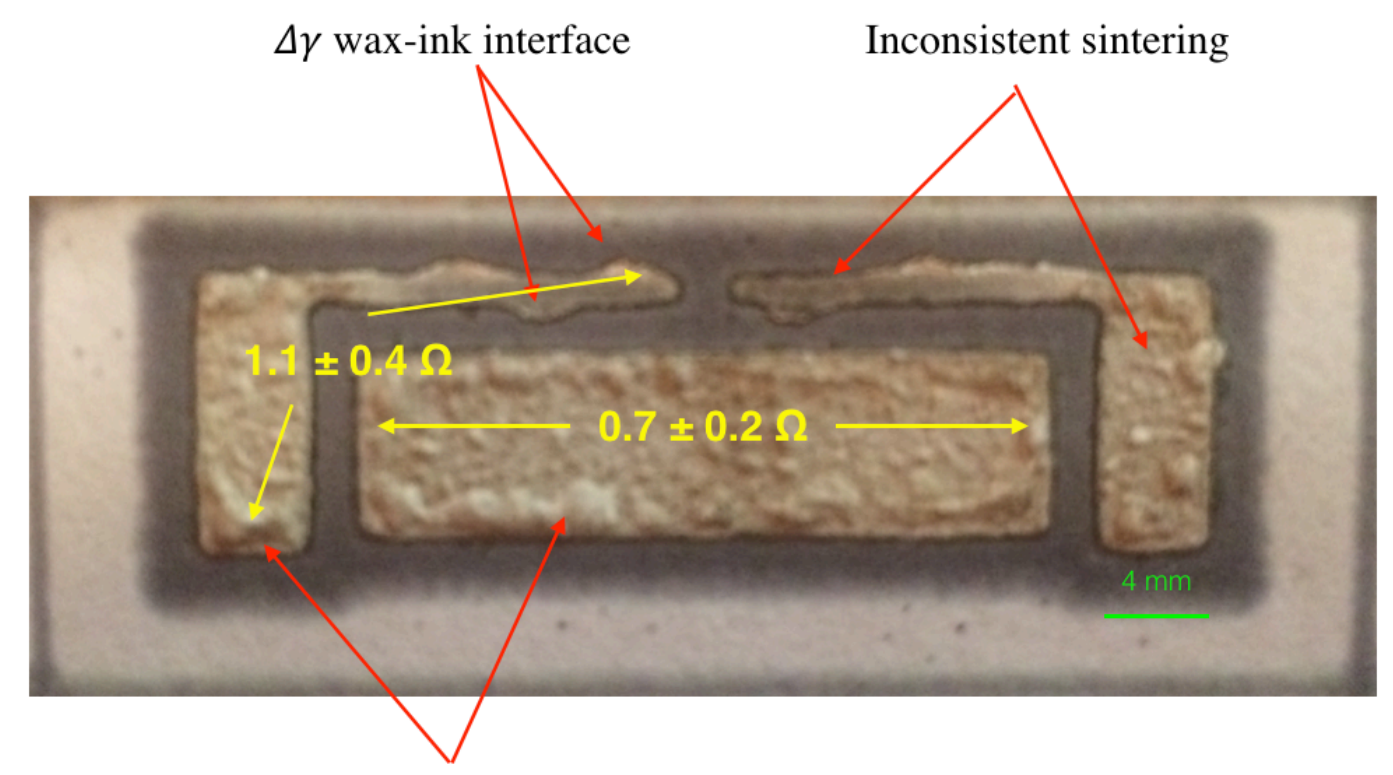

Uneven solvent evaporation

Figure 10. Sintered circuitry layer

Several failure modes were present upon sintering: loss of definition in wax-printed channels, inconsistent sintering in narrow-channel sections of the side traces and uneven solvent evaporation resulting in thickness variations. Possible causes of these phenomena are discussed in later sections.

\subsection{Flexibility}

Fatigue is a primary failure mode of flexible printed media and therefore a strong metric for a material's candidacy. Electrical conduction is the primary functionality of the ink, so characterizing its performance under the primary failure condition is the most appropriate - hence the comparison of resistivity against fold angle is salient.

Fold angle is defined as acute when the circuitry is folded against itself, relative to the printed face. Obtuse folding occurs in the opposite direction, or in a "backwards" fashion 
relative to the printed face (Siegel et al., 2010). (Figure 11) Samples were prepared by printing $0.636 \mu \mathrm{L} \cdot \mathrm{mm}^{-3}$ of conductive ink on $8 \times 2.75 \times 0.18 \mathrm{~mm}$ wax-printed traces, then sintered at $150^{\circ} \mathrm{C}$ for 30 minutes. Resistance measurements were taken by folding samples along $90^{\circ}$ and $180^{\circ}$ edges of a machined insulator, namely polyvinyl chloride (PVC). A minimum two-fold increase in resistance was observed upon folding, regardless of angle or direction. Increases as high as $1500 \%$ were observed in the obtuse condition, indicating behavior sensitive to directionality, where unequal changes in resistance were observed in fold angle whether acute $\left(-180^{\circ}\right.$ to $\left.0^{\circ}\right)$ or obtuse $\left(0^{\circ}\right.$ to $\left.180^{\circ}\right)$.

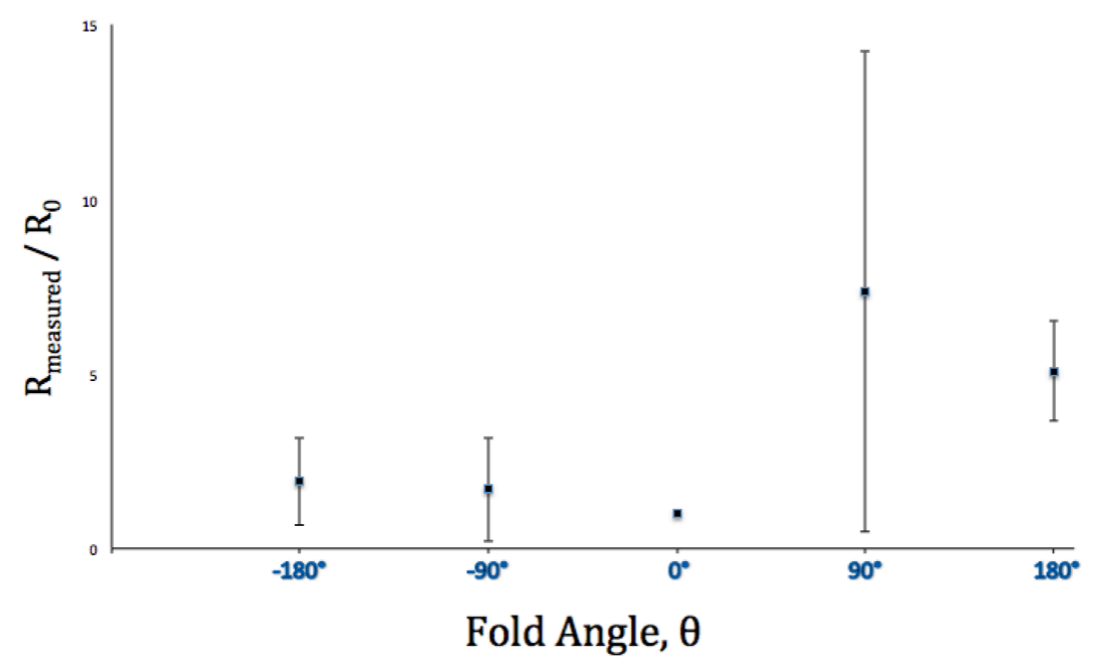

Figure 11. Resistivity of circuitry traces with respect to fold angle

\subsection{X-ray Diffraction}

X-ray diffraction patterns were collected using a Siemens Diffraktometer D5000. A scan was taken ranging from $10-140^{\circ} 2 \theta$ in $0.1^{\circ}$ increments at a rate of $10^{\circ}$ per minute. As a baseline, the diffraction pattern of layer 8 with wax traces but without conductive ink 
applied ("empty") was collected. (Figure 12) shows two high-intensity, low-angle, broad peaks often corresponding with semi-crystalline domains of cellulose I. The characteristic double-peak at $14.8^{\circ}$ and $16.5^{\circ}(2 \theta)$ correspond to the (101) plane reflection of cellulose I, while the narrow, intense peak at $22.6^{\circ} 2 \theta$ corresponds to (002). A third, shallow signal found at $34.4^{\circ} 2 \theta$ matches the (004) reflection; slower scan speeds could extract additional resolution to this peak. Relative intensities, locations, and widths strongly match literature. (Costa et al., 2014; Veigas et al., 2012)

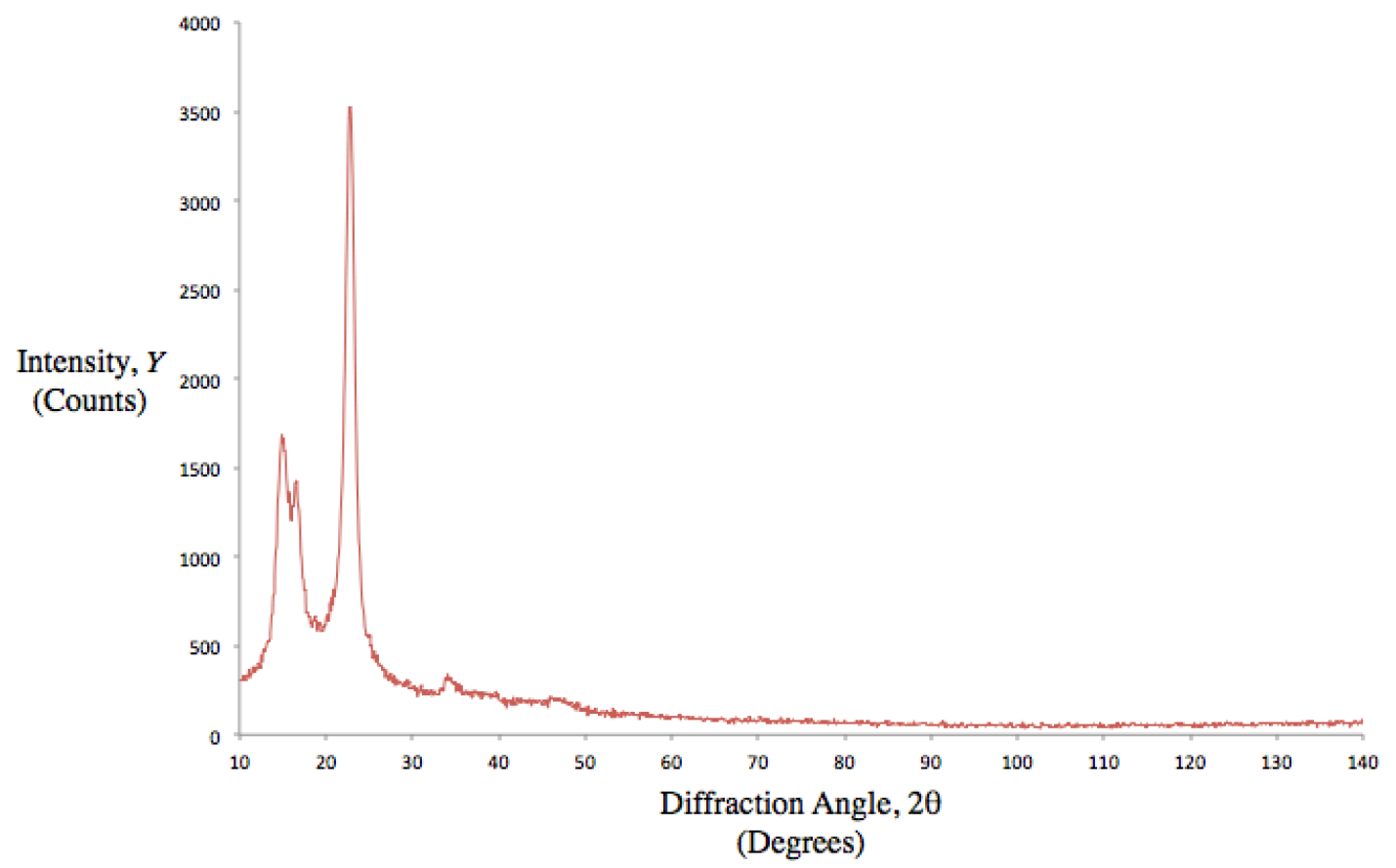

Figure 12. Diffraction pattern of unprinted circuitry layer

It must be noted that peaks for solid wax were not observed in the diffraction pattern but were present in the sample tested; it is likely the mounting configuration can be rearranged to include wax traces within the detector's scan area. 
Immediately following testing of the "empty" layer, the diffraction pattern was collected under identical conditions for a layer-8 sample with the conductive ink printed and sintered. (Figure 13) The tested sample was treated with the highest volumetric ink density of $0.5 \mu \mathrm{L} \cdot \mathrm{mm}^{-3}$.

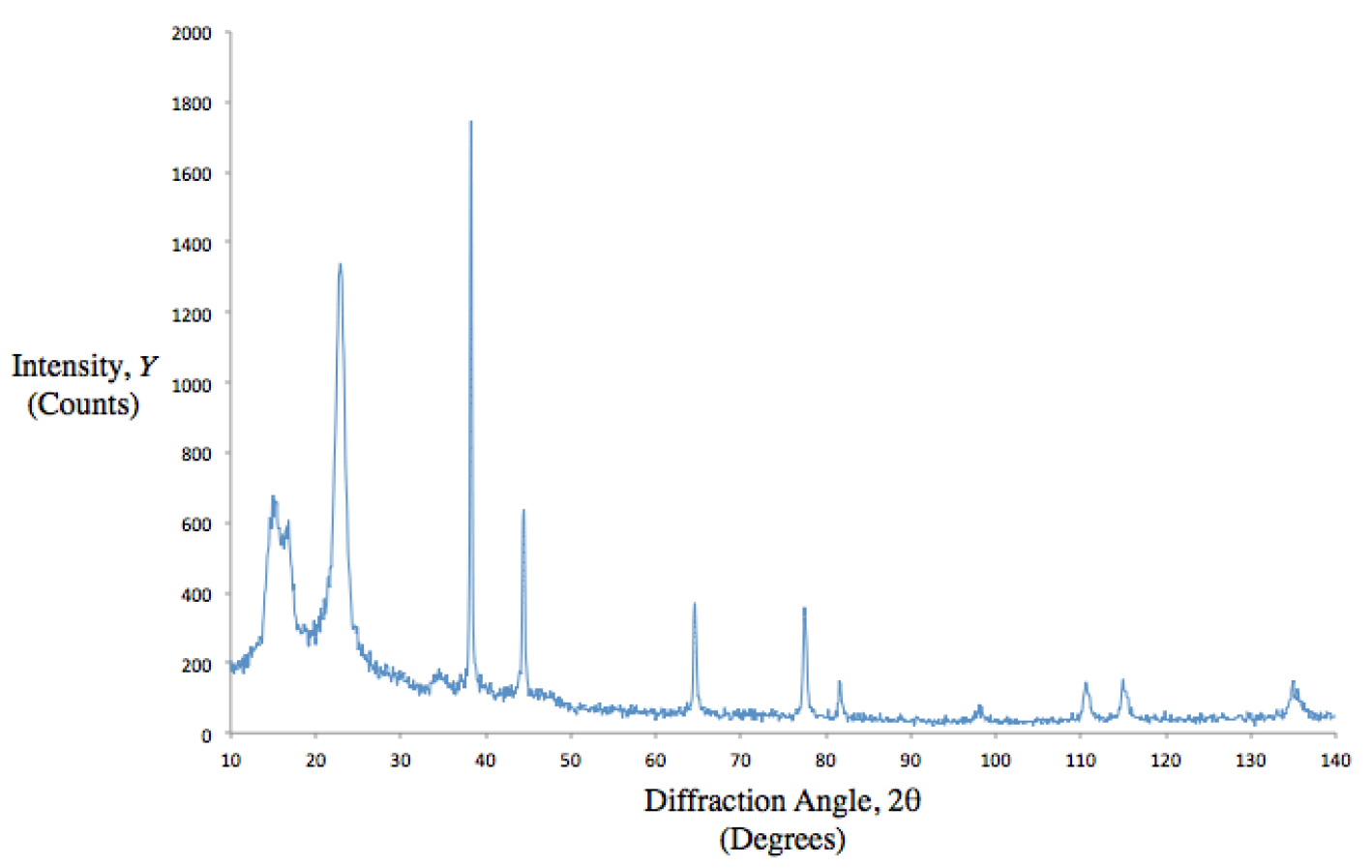

Figure 13. Diffraction pattern of sintered circuitry layer

The characteristic peaks of cellulose 1 were again present but with intensities lower relative to the numerous narrow peaks typical of close-packed lattices. Given that pure solid silver exhibits face-centered cubic symmetry, it is no surprise the (111) plane reflection is present at $38.3^{\circ} 2 \theta$ and strongest. Additional reflections [i.e. (200), (220), (311), (222)] at their respective locations (i.e. $44.5^{\circ}, 64.6^{\circ}, 77.5^{\circ}, 81.7^{\circ} 2 \theta$ ) only strengthen the conclusion that bulk silver was formed after sintering. Additionally, the 
absence of peaks corresponding to silver acetate indicates complete sintering of supernatant (Theivasanthi \& Alagar, 2012; Walker \& Lewis, 2012; Young, 1975).

\subsection{Conductivity Threshold}

Conductivity threshold was mapped by measuring resistance of a given trace volume when sinter time, temperature, and ink concentration are varied. To conserve ink precursor and simplify the test matrix a $150^{\circ} \mathrm{C}$ isothermal-hold sintering profile was chosen for preliminary testing. (Figure 14) Sintering times above 30 minutes exhibit highest sensitivities at moderate concentrations, approximately $0.5 \mu \mathrm{L} \cdot \mathrm{mm}^{-3}$, but insensitive elsewhere. The ink concentration, however, was observed to decrease resistivity exponentially above $0.3 \mu \mathrm{L} \cdot \mathrm{mm}^{-3}$, regardless of time. It must be noted that empty cells in the matrix correspond to data points that were omitted due to either unstable readings, compromised deposition, or sample damage.

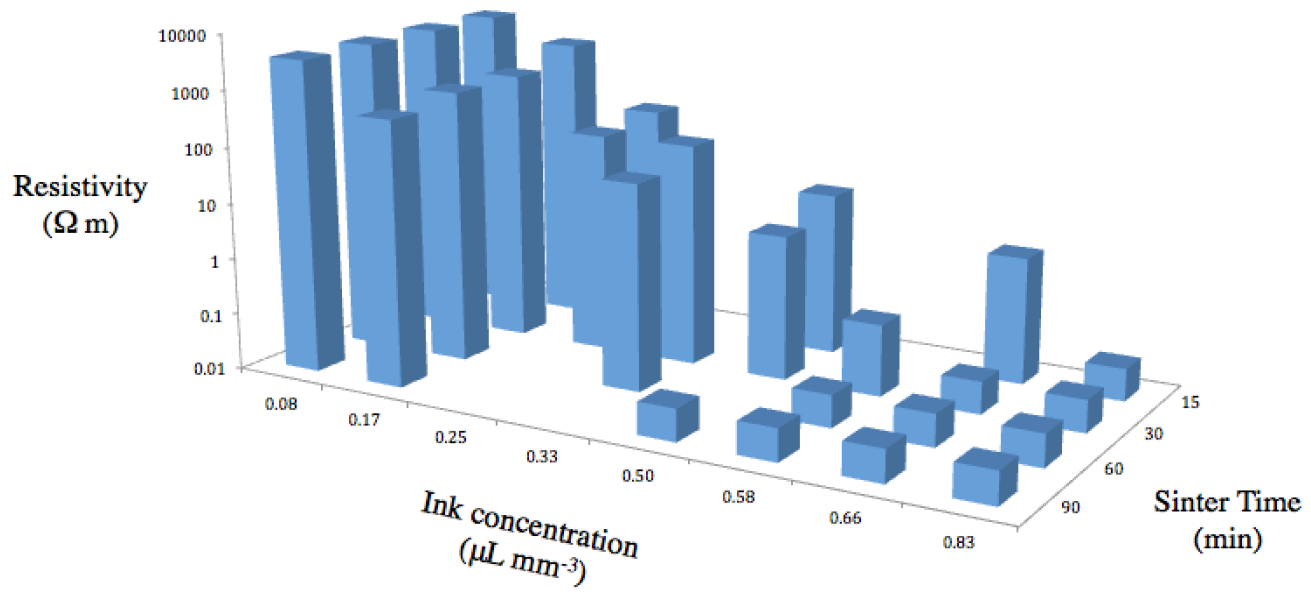

Figure 14. Two-factor matrix of conductivity threshold 
Upon identifying a narrower range of ink concentrations and sinter times at which an operating limit for ink concentration could be established, another test matrix was conducted to include variations in sinter temperature. (Figure 15) While a temperature increase appeared to have larger effect at low concentration (i.e. $0.36 \mu \mathrm{L} \cdot \mathrm{mm}^{-3}$ ), the impact was observed to diminish above $0.5 \mu \mathrm{L} \cdot \mathrm{mm}^{-3}$. Conversely, at higher temperatures $\left(150^{\circ} \mathrm{C}\right)$, the effect of increasing concentration has little impact on resistivity. In this case, it may be possible that labile amine ligand evaporation occurred sooner in the heat treatment and thus the concentration sensitivity is not visible.

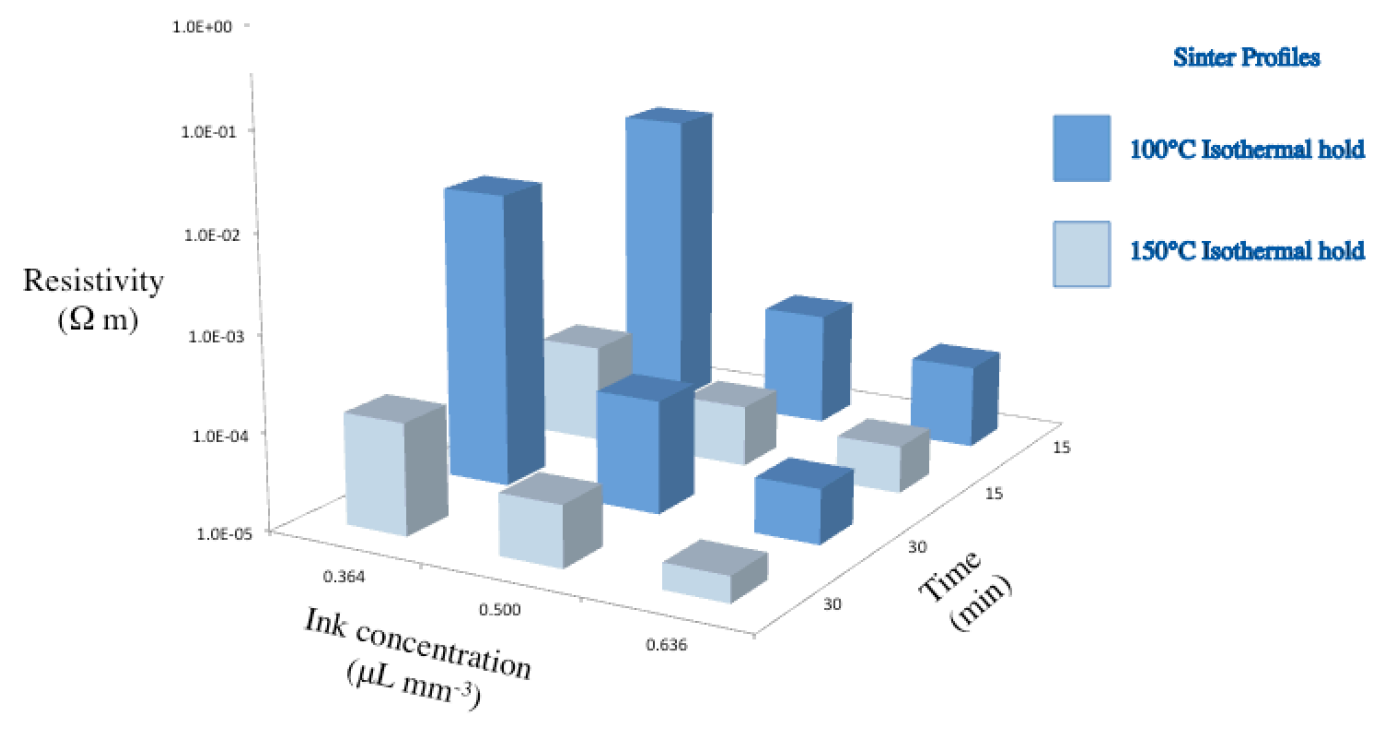

Figure 15. Three-factor matrix of conductivity threshold 


\subsection{Battery Performance}

\subsubsection{Open-circuit Voltage}

Open circuit voltage $\left(\mathrm{V}_{\mathrm{OC}}\right)$ of a representative sample device was sustained for $40+$ minutes at $2.5 \mathrm{~V}$ with less than $10 \%$ degradation after 70 minutes. (Figure 16) Although performance of each cell (approximately 1.2 $-1.3 \mathrm{~V}$ ) is poor in relation to the theoretical potential $(2.4 \mathrm{~V})$, the performance agrees with the reported voltage profile of the original authors. The profile of a failed device is also plotted to illustrate the behavior of a device with failure in one cell. A short explains the delayed increase and subsequent sudden decrease to approximately half the peak voltage. Measurement of a zero voltage across that cell confirms the short; measurement of the other cell accounts for approximately all $1.3 \mathrm{~V}$.

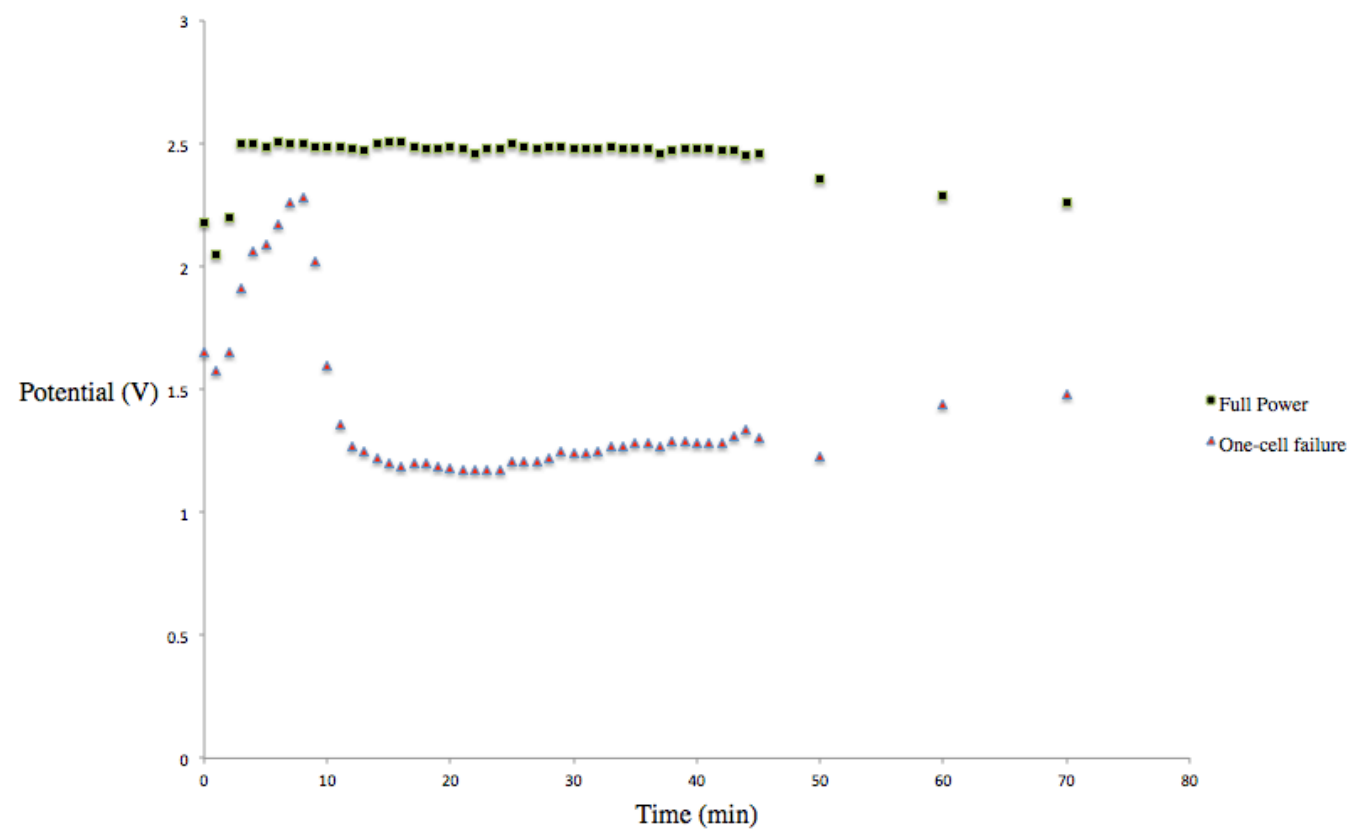

Figure 16. Open-circuit voltage profile 


\subsubsection{Short-circuit Current}

Short-circuit current $\left(\mathrm{I}_{\mathrm{SC}}\right)$ measurements were taken using an ammeter and $1.5 \Omega$ load resistor, both in series with the fluidic battery. The current profile of the three best performing devices were plotted to represent ideal current profile. (Figure 17) The sharp increase in current at approximately 3-6 minutes indicates the reconstitution of salt bridges and "turn on" of the battery. The convergence of values at 20-25 minutes indicates a cessation of the reduction-oxidation reaction generating galvanic power. The full-duration half-max time (16 minutes, $200 \mu \mathrm{A})$ again correlates strongly with the original battery design.

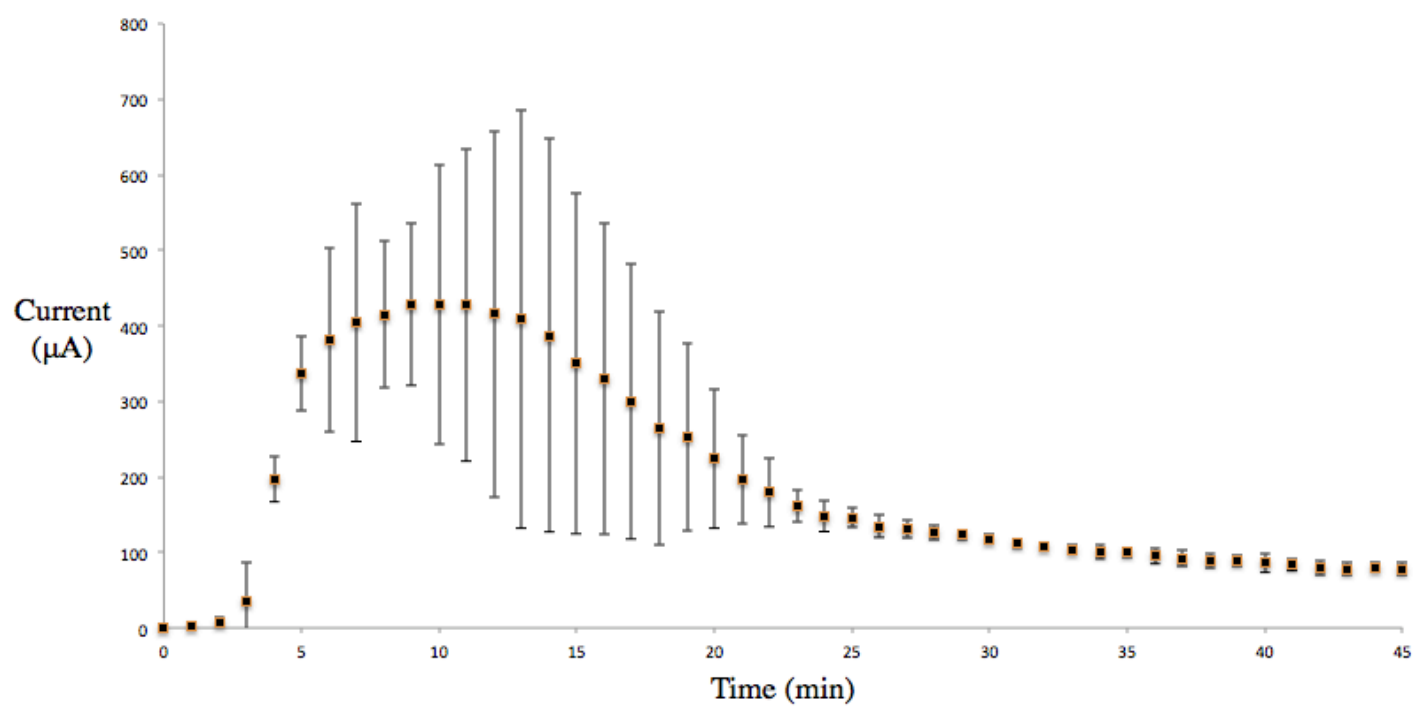

Figure 17. Mean short-circuit current profile 


\section{DISCUSSION}

\subsection{Resistivity}

It is expected that the cellulose substrate creates significant electron scattering, but so far only empirical or qualitative relationships have been determined between pore characteristics and metal nanoparticle-patterned circuitry (Hsieh, Kim, Nogi, \& Suganuma, 2013). In fact, the resistivity of cellulose 1 itself is heavily dependent on fiber direction, pore network, impurity or solvent concentration, and temperature, ranging from $10^{9}$ to $10^{10} \Omega \cdot \mathrm{m}$ at ambient conditions (Takahashi \& Takenaka, 1983). Additionally, there is an order of magnitude change in resistivity for silver at film thicknesses below $500 \mathrm{~nm}$, but it appears an unlikely influence given the deposition volume (Lacy, 2011). Given the results, it is inferred that the cellulose has an appreciable effect on electron scattering within the final circuitry. The characterization of relative influence between cellulose and silver on electron mobility is outside the scope of this paper but would be insightful for further development. The loss of definition of wax-printed channels is accounted for by a change in surface energy at the ink-wax interface. Again, this reaction is multi-faceted and beyond the scope of this study, but it is clear that elevated sinter temperatures provide favorable kinetics for this reaction as it is less prevalent at $100^{\circ} \mathrm{C}$ isothermal holds. The inconsistent sintering and uneven thickness can be attributed to the concentration gradient of nanoparticles adhered to the cellulose pore network. These variations may also compound the failure modes during folding.

A large error in resistivity is attributed to the poor resolution of thickness measurements. This error can be estimated by differentiating the resistance model used with respect to cross-sectional thickness (Equation 6), 


$$
\begin{aligned}
& d \rho=\frac{R d A}{l} \\
& d \rho=\frac{R w}{l} d t
\end{aligned}
$$

Given a $0.05 \mathrm{~mm}$ resolution in thickness measurements, an estimated resistivity error of $5.8610^{-6} \Omega \cdot \mathrm{m}$ is calculated. However, despite this range of error, it does not bridge the order-of-magnitudes difference between the experimental and ideal resistivity values. Scanning electron microscopy and profilometry should be used to identify the range of trace thickness with more resolution and thus narrow this error.

\subsection{Flexibility}

A key advantage of conductive ink during material selection was the symmetry of electrical properties regardless of which side experienced deposition. The silver nanoparticles were assumed to embed with the porous cellulose network omnidirectionally. By comparison, printed metal wires do not share this symmetry and are thus sensitive to fold directionality (Siegel et al., 2010). It appears this symmetry advantage is also not present using this ink as circuitry, most likely attributable to the variation in grain structure. Unpredictable dispersion of nanoparticles during printing creates a non-uniform grain structure after sintering. Subsequently, the debonding, channeling, and surface-crack failure modes found often in thin-film structures would explain the poor fatigue characteristics in this circuitry material's non-uniform, poorly sintered grain structures (Hutchinson, 1996). It has been speculated that, due to the differences in compressive and tensile stresses of bend directionality, the small cracks formed during acute folding do not fracture the entire percolating network, while the large cracks from obtuse bending create creases that sever the electrical connection 
between sides (Siegel et al., 2010). Scanning electron microscopy may also provide insight regarding the nanoparticle adhesion variations within the pore network, grain structures after sintering, and failure modes present during folding.

\subsection{X-ray Diffraction}

The diffraction patterns provide overwhelming confidence in the formation of bulk silver after sintering. The narrow full-width half-max of the metallic peaks indicate high crystallinity, which implies a well-sintered Ag-nanoparticle network (Song et al., 2014). The relative intensities of reflections between the cellulose and silver planes served as an interesting indicator of the structural and surface changes in the substrate, but do not translate to weighted fractions of the hybrid conductive network. Considering resistivity is dependent on this metal-porous media interaction, $\mathrm{x}$-ray diffraction would again serve as an integral tool for mapping the relationship between volumetric particle density deposited and electrical performance.

\subsection{Conductivity Threshold}

There are three competing factors influencing the conductivity threshold of the printed ink. Firstly, A minimum concentration of dispersed nanoparticles defines the percolation threshold, below which electrical conduction and atomic diffusion are precluded. As evidenced by the invariant resistivity of all $0.8 \mu \mathrm{L} \cdot \mathrm{mm}^{-3}$ samples, the printed circuitry is insensitive to both high sinter temperature and extended sinter times. In conditions with low dispersion rates of nanoparticles after printing (i.e. low ink concentrations), the lack of a percolating network precludes electron conduction. 
Secondly, a minimum temperature defines the percolation temperature, above which the evaporation of amine ligands is possible. It has been established that the minimum temperature of percolation is heavily dependent on the evaporation of labile nanoparticle ligands (Anto, Sivaramakrishnan, Chua, \& Ho, 2010). While on polymer substrates the Ag-nanoparticle conductive ink used exhibited an evaporation temperature of $90^{\circ} \mathrm{C}$, preliminary sintering tests showed minimal change until above $100^{\circ} \mathrm{C}$. At temperatures above this threshold, the printed circuitry was conductive and only sensitive to treatment time and ink concentration. Therefore, it can be assumed that the conductivity threshold for this system (ink on cellulose) is also determined by the thermal stability of the amine ligands within the cellulose pore network. Studying the absorption profile of this ink using Fourier-transform infrared spectroscopy while varying exposure to different sinter times and temperatures could identify the evaporation temperature more accurately.

Thirdly, upon the formation of a percolating network of sufficiently connected dispersed nanoparticles, atomic diffusion is possible and sintering can occur. Given the reduced melting temperatures of metallic nanoparticles and homologous temperature dependence on diffusion-mechanism, extended heating $\left(150^{\circ} \mathrm{C}\right.$ for $30+$ minutes $)$ may experience a minimal but non-trivial shift into the densification regime of sintering. The reduction of stacking fault densities during densification translates to reduced electron scattering, thus increasing conductivity. Such coalescence methods of aggregated nanoparticles are evident in similar metallic systems (i.e. Au) but not yet explored on cellulose substrates (Ingham et al., 2011). This could account for the final decrease in resistivity below $10^{-4}$ $\Omega \cdot m$ in samples with highest concentrations, times, and temperatures. Profilometry of 
high-temperature long-exposure samples could be used to confirm the change in trace volume.

\subsection{Battery Performance}

\subsubsection{Open-circuit Voltage}

The equipment manufacturer specifies a maximum instrumental error of $\pm 0.2 \mathrm{~V}$ in potential measurements (Innova Electronic Corporation, 2016). This error accounts for $8 \%$ of the measured value of the devices' peak performance $(2.5 \mathrm{~V})$. However, the requisite voltage threshold of $1.8 \mathrm{~V}$ for ultraviolet LEDs is more than $25 \%$ below the microfluidic battery performance and thus does not reduce confidence in satisfying the functional requirement.

\subsubsection{Short-circuit Current}

A maximum instrumental error of $\pm 1.5 \%$ is specified for ultra-low current measurements. Short-circuit current was used to determine "on" time, defined as the fullduration half-max of the current profile. This error in current measurement translates to a \pm 0.88 minute error in FDHM, a trivial impact on power generation when satisfying the functional requirement. 


\section{CONCLUSION}

\subsection{Measuring Success}

The purpose of defining unambiguous testable system requirements is to measure the success of the hypothesis in quantitative terms. (Table 2) The circuitry material was specified to be both conduct and stable; the low absolute resistance of the circuitry was dwarfed by the internal resistance of the battery and experienced no change no noticeable changed over time. The integrated circuitry was specified to preserve both robust flexibility and mild temperatures; while the circuitry can be sintered successfully using a $150^{\circ} \mathrm{C}$ isothermal, the fatigue during folding failed to perform with the flexibility of competing approaches. The platform was specified to provide sufficient data collection time and power to interfacing components. The full-duration half-max of power generation was 16 minutes, far exceeding the requisite 5 minutes. The threshold voltage and forward current minimums of commercially available LEDs were both exceeded. 
Table 2. Success meeting system requirements

\begin{tabular}{|c|c|c|c|}
\hline $\begin{array}{c}\text { System } \\
\text { Requirement }\end{array}$ & $\begin{array}{l}\text { Functional } \\
\text { Requirement }\end{array}$ & $\begin{array}{l}\text { Quantitative } \\
\text { Metric }\end{array}$ & $\begin{array}{l}\text { Success } \\
\text { Achieved }\end{array}$ \\
\hline Conductive & $<5 \%$ power loss & $\begin{array}{l}<1.5 \Omega \text { absolute } \\
<0.1 \% \mathrm{R}_{\text {circuit }} / \mathrm{R}_{\text {internal }}\end{array}$ & Yes \\
\hline Stable & Stable at STP & $\begin{array}{c}\Delta \rho=0 \text { over } 30 \\
\text { days }\end{array}$ & Yes \\
\hline Flexible & $<20 \% \Delta \rho$ at $90^{\circ}$ & $>200 \% \Delta \rho$ at $90^{\circ}$ & No \\
\hline Low-temperature & All $\mathrm{T}<200^{\circ} \mathrm{C}$ & $\begin{array}{c}150^{\circ} \mathrm{C}, \\
0.636 \mu \mathrm{L} \cdot \mathrm{mm}^{-3}\end{array}$ & Yes \\
\hline Data collection & $>5$ minutes "on" time & FDHM 16 minutes & Yes \\
\hline Interface-able & $>1.8 \mathrm{~V}, 100 \mu \mathrm{A}$ & $2.4 \mathrm{~V}, 200 \mu \mathrm{A}$ & Yes \\
\hline
\end{tabular}

\subsection{Future Design}

A future design for an automatable all-printed was developed to incorporate features new to the $\mu \mathrm{PAD}$ industry. (Figure 18) The advent of "origami" folding features opens interesting solutions to assembly stages that are otherwise inaccurate or poorly suited for automation. The proposed design can be automated with some processes in parallel, such as electrolyte deposition and ink application. Furthermore, it is well suited for printing and assembly in replicates. 


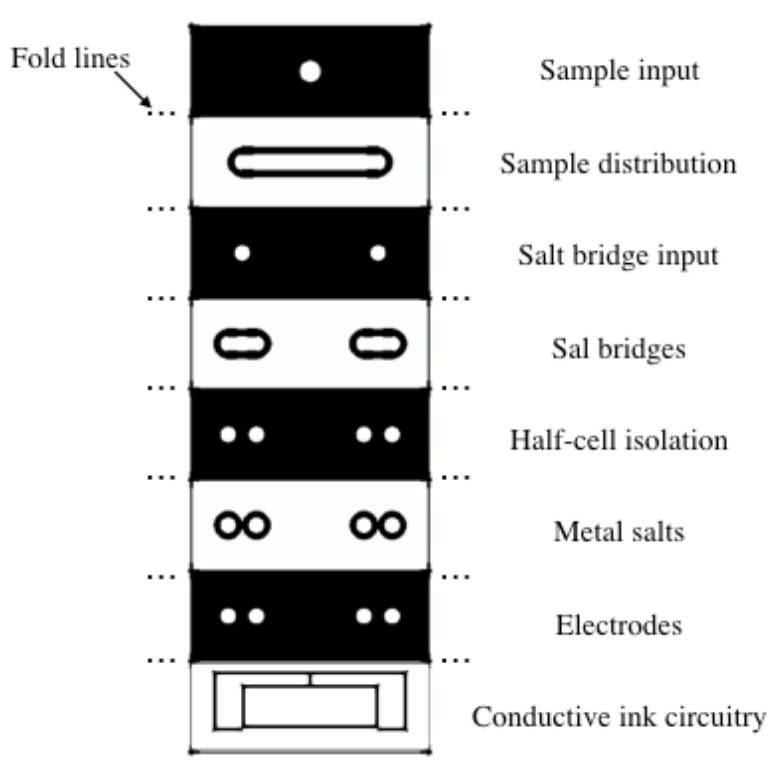

Figure 18. Future all-printed platform design

Several additional design features can be added to increase functionality of the platform or reduce the assembly errors:

- Alignment reticles can be added for assembly quality control as layer count increases.

- Electrode pad shapes or colors can differ between metals to ensure assembly accuracy.

- Use multiple electrode metals to create various voltage levels that accommodate diverse needs of electrical components. 


\section{BIBLIOGRAPHY}

3M Corporation. (2014). 200MP 3M ${ }^{\mathrm{TM}}$ Adhesive Transfer Tapes with Adhesive 200MP.

Adkins, J., Boehle, K., \& Henry, C. (2015). Electrochemical paper-based microfluidic devices. Electrophoresis, 36(16), 1811-1824. http://doi.org/10.1002/elps.201500084

Allen, G. L., Bayles, R. A., Gile, W. W., \& Jesser, W. A. (1986). Small particle melting of pure metals. Thin Solid Films, 144(2), 297-308. http://doi.org/http://dx.doi.org/10.1016/0040-6090(86)90422-0

Anto, B. T., Sivaramakrishnan, S., Chua, L. L., \& Ho, P. K. H. (2010). Hydrophilic sparse ionic monolayer-Protected metal nanoparticles: Highly concentrated nano-Au and nano-Ag "Inks" that can be sintered to near-Bulk conductivity at $150 \mathrm{C}$. Advanced Functional Materials, 20(2), 296-303.

http://doi.org/10.1002/adfm.200901336

Asoro, M. A., Damiano, J., \& Ferreira, P. J. (2009). Size Effects on the Melting Temperature of Silver Nanoparticles : In-situ TEM Observations, 15(Suppl 2), 2391-2393. http://doi.org/10.1017/S1431927609097013

Carrilho, E., Martinez, A. W., \& Whitesides, G. M. (2009). Understanding wax printing: A simple micropatterning process for paper-based microfluidics. Analytical Chemistry, 81(16), 7091-7095. http://doi.org/10.1021/ac901071p

Cate, D. M., Adkins, J. A., Mettakoonpitak, J., \& Henry, C. S. (2015). Recent developments in paper-based microfluidic devices. Analytical Chemistry, 87(1), 1941. http://doi.org/10.1021/ac503968p

Christensen, K. (2002). Percolation theory. Thesis, 1-40.

Costa, M. N., Veigas, B., Jacob, J. M., Santos, D. S., Gomes, J., Baptista, P. V , .. 
Fortunato, E. (2014). A low cost, safe, disposable, rapid and self-sustainable paperbased platform for diagnostic testing: lab-on-paper. Nanotechnology, 25(9), 94006. http://doi.org/10.1088/0957-4484/25/9/094006

Defense Acquisition University Press. (2001). Systems Engineering Fundamentals.

Deptuck, D., Harrison, J. P., \& Zawadzki, P. (1985). Measurement of elasticity and conductivity of a three-dimensional percolation system. Phys. Rev. Lett., 54(9), 913916. http://doi.org/10.1103/PhysRevLett.54.913

Ellerbee, A. K., Phillips, S. T., Siegel, A. C., Mirica, K. A., Martinez, A. W., Striehl, P., ... Whitesides, G. M. (2009). Quantifying colorimetric assays in paper-based microfluidic devices by measuring the transmission of light through paper. Analytical Chemistry, 81(20), 8447-8452. http://doi.org/10.1021/ac901307q

Ferry, D., Goodnick, S., \& Bird, J. (2009). Transport in Nanostructures (Second). Cambridge University Press.

George, S., \& Sebastian, M. T. (2008). Effect of sintering temperature on the percolation threshold and the dielectric. http://doi.org/10.1016/j.compscitech.2008.04.022

Glavan, A. C., Walker, S. B., Lewis, J. A., Whitesides, G. M., \& Link, C. (2016). Inkjet Printing of Conductive Inks with High Lateral Resolution on Omniphobic “ R F Paper" for Paper-Based Electronics and MEMS.

Greer, J. R., \& Street, R. A. (2007). Thermal cure effects on electrical performance of nanoparticle silver inks, 55, 6345-6349. http://doi.org/10.1016/j.actamat.2007.07.040

Hazmat2. (2012). Galvanic cell, labeled. Retrieved January 12, 2016, from https://commons.wikimedia.org/wiki/File:Galvanic_cell_labeled.svg 
Heitjans, P., \& Kärger, J. (2005). Diffusion in condensed matter: Methods, materials, models. Diffusion in Condensed Matter: Methods, Materials, Models. http://doi.org/10.1007/3-540-30970-5

Holm, E. A., \& Cima, M. J. (1989). Two-Dimensional Whisker Percolation in Ceramic Matrix-Ceramic Whisker Composites. Journal of the American Ceramic Society, 72(2), 303-305. http://doi.org/10.1111/j.1151-2916.1989.tb06119.x

Hsieh, M., Kim, C., Nogi, M., \& Suganuma, K. (2013). Electrically conductive lines on cellulose nanopaper for fl exible electrical devices, 9289-9295. http://doi.org/10.1039/c3nr01951a

Hutchinson, J. W. (1996). Stresses and Failure Modes in Thin Films and Multilayers. Thin Films.

Ingham, B., Lim, T. H., Dotzler, C. J., Henning, A., Toney, M. F., \& Tilley, R. D. (2011). How nanoparticles coalesce: An in situ study of Au nanoparticle aggregation and grain growth. Chemistry of Materials, 23(14), 3312-3317.

http://doi.org/10.1021/cm200354d

Innova Electronic Corporation. (2016). Innova 3320 Auto-ranging Digital Multi-meter Product Specifications.

Kim, D., \& Moon, J. (2005). Highly Conductive Ink Jet Printed Films of Nanosilver Particles for Printable Electronics, 45-48. http://doi.org/10.1149/1.2073670

Knoerr, M., Kraft, S., \& Schletz, A. (2010). Reliability assessment of sintered nano-silver die attachment for power semiconductors. In 2010 12th Electronics Packaging Technology Conference, EPTC 2010 (pp. 56-61). http://doi.org/10.1109/EPTC.2010.5702605 
Lacy, F. (2011). Developing a theoretical relationship between electrical resistivity, temperature, and film thickness for conductors. Nanoscale Research Letters, 6(1), 636. http://doi.org/10.1186/1556-276X-6-636

Lan, W.-J., Maxwell, E. J., Parolo, C., Bwambok, D. K., Subramaniam, A. B., \& Whitesides, G. M. (2013). Paper-based electroanalytical devices with an integrated, stable reference electrode. Lab on a Chip, 13(20), 4103-8. http://doi.org/10.1039/c3lc50771h

LITEON Optoelectronics. (2006). SMD LED Product Data Sheet. LITE-ON Technology Corp.

Park, T. S., Li, W., McCracken, K. E. K., \& Yoon, J. J.-Y. (2013). Smartphone quantifies Salmonella from paper microfluidics. Lab on a Chip, 22(24), 256-258. http://doi.org/10.1039/c3lc50976a

Perelaer, J., Abbel, R., Wünscher, S., Jani, R., \& Lammeren, T. Van. (2012). Roll-to-Roll Compatible Sintering of Inkjet Printed Features by Photonic and Microwave Exposure : From Non-Conductive Ink to ... Roll-to-Roll Compatible Sintering of Inkjet Printed Features by Photonic and Microwave Exposure : From NonConductive Ink to , (May). http://doi.org/10.1002/adma.201104417

Rahaman, M. . (2003). Ceramic Processing and Sintering.

Shari, F., Ghobadian, S., Cavalcanti, F. R., \& Hashemi, N. (2015). Paper-based devices for energy applications, 52, 1453-1472. http://doi.org/10.1016/j.rser.2015.08.027

Siegel, B. A. C., Phillips, S. T., Dickey, M. D., Lu, N., Suo, Z., \& Whitesides, G. M. (2010). Foldable Printed Circuit Boards on Paper Substrates, 28-35. http://doi.org/10.1002/adfm.200901363 
Song, J. M., Chen, W. T., Hsieh, K. H., Kao, T. H., Chen, I. G., Chiu, S. J., \& Lee, H. Y. (2014). An in situ study on the coalescence of monolayer-protected Au-Ag nanoparticle deposits upon heating. Nanoscale Res Lett, 9(1), 438. http://doi.org/10.1186/1556-276X-9-438

Takahashi, M., \& Takenaka, H. (1983). DC electrical conductivity of cellulose. Polymer Journal. http://doi.org/10.1295/polymj.15.625

Tanner, D. B., \& Larson, D. C. (1968). Electrical Resistivity of Silver Films.pdf. Phys. Rev., 166(3). http://doi.org/https://doi.org/10.1103/PhysRev.166.652

Theivasanthi, T., \& Alagar, M. (2012). Electrolytic Synthesis and Characterizations Of Silver Nanopowder. Nano Biomedicine and Engineering, 4, 1-11. http://doi.org/10.5101/nbe.v4i2.p58-65

Thom, N. K., Lewis, G. G., DiTucci, M. J., \& Phillips, S. T. (2013). Two general designs for fluidic batteries in paper-based microfluidic devices that provide predictable and tunable sources of power for on-chip assays. RSC Advances, 3(19), 6888-6895. http://doi.org/10.1039/C3RA40701B

Thom, N. K., Lewis, G. G., DiTucci, M. J., \& Phillips, S. T. (2013). Two general designs for fluidic batteries in paper-based microfluidic devices that provide predictable and tunable sources of power for on-chip assays. RSC Advances, 3(19), 6888. http://doi.org/10.1039/c3ra40701b

Thom, N. K., Yeung, K., Pillion, M. B., \& Phillips, S. T. (2012). "Fluidic batteries” as low-cost sources of power in paper-based microfluidic devices. Lab on a Chip, 12(10), 1768. http://doi.org/10.1039/c2lc40126f

Veigas, B., Jacob, J. M., Costa, M. N., Santos, D. S., Viveiros, M., Inácio, J., ... Baptista, 
P. V. (2012). Gold on paper-paper platform for Au-nanoprobe TB detection. Lab on a Chip, 12(22), 4802-8. http://doi.org/10.1039/c21c40739f

Walker, S. B., \& Lewis, J. A. (2012). Supporting Information - Reactive Silver Inks for Patterning High-Conductivity Features at Mild Temperatures, (I), 1-6. http://doi.org/10.1021/ja209267c

Wronski, C. R. M. (2002). The size dependence of the melting point of small particles of tin. British Journal of Applied Physics, 18(12), 1731-1737.

http://doi.org/10.1088/0508-3443/18/12/308

Young, D. A. (1975). Phase diagrams of the elements. Lawrence Livermore Laboratory, 64. http://doi.org/10.2172/4010212 


\section{APPENDICES}

Appendix A

Layer 1

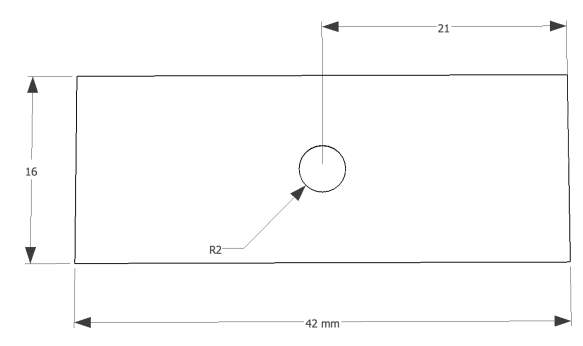

Layer 3

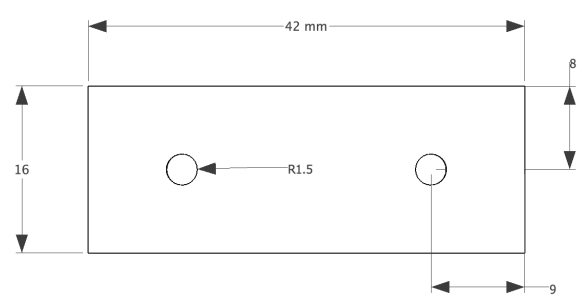

Layer 5

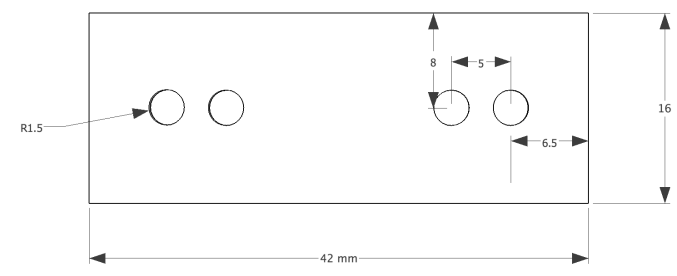

Layer 7

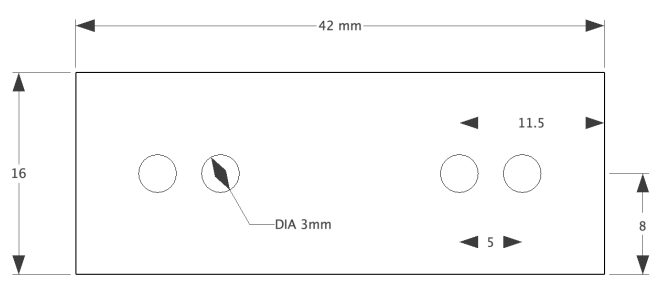

Layer 2

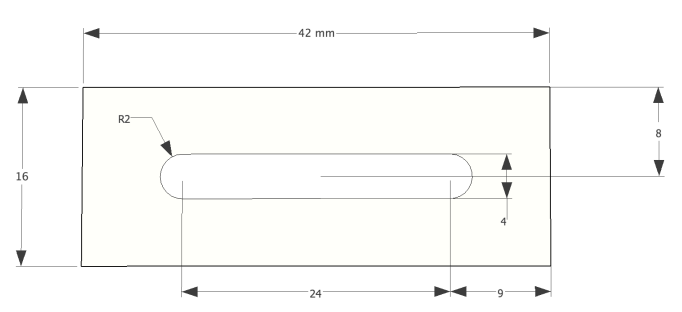

Layer 4

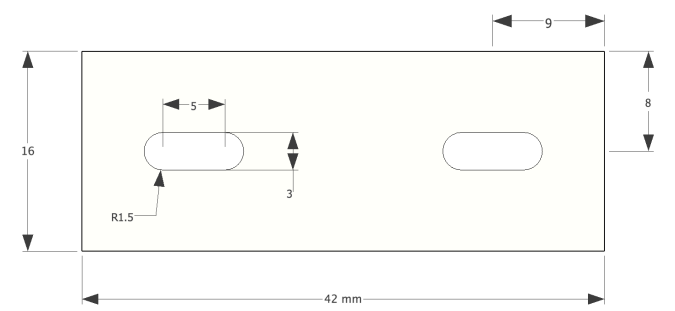

Layer 6

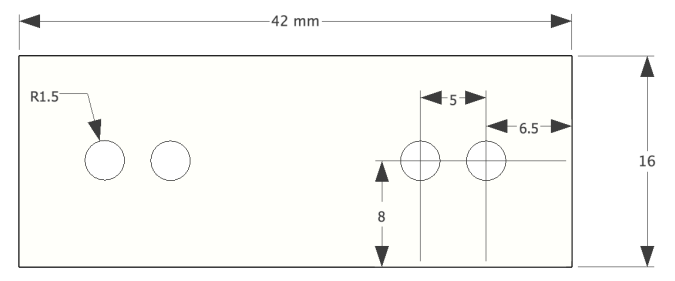

Layer 8

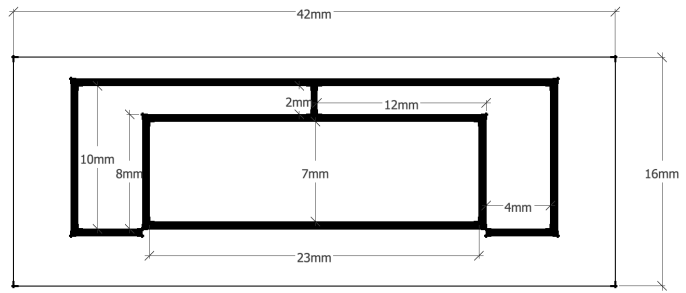


Appendix B

Table 3. Bill of materials

\begin{tabular}{|c|c|c|c|}
\hline Material & Amount & Price & Specifications \\
\hline $\mathrm{AlCl}_{3}$ & $100 \mathrm{~g}$ & $\overline{\$ \$ 58.40}$ & ReagentPlus® \\
\hline $\mathrm{AgNO}_{3}$ & $5 \mathrm{~g}$ & $\$ 29.40$ & ReagentPlus ${ }^{\circledR}$, \\
\hline $\mathrm{NaNO}_{3}$ & $5 \mathrm{~g}$ & $\$ 39.80$ & ReagentPlus ${ }^{\circledR}$, \\
\hline $\mathrm{Al}(\mathrm{s})$ & $50 \times 50 \mathrm{~mm}$ & $\$ 55.40$ & $\begin{array}{l}99.999 \% \text { pure, } \\
0.25 \mathrm{~mm} \text { foil }\end{array}$ \\
\hline $\operatorname{Ag}(\mathrm{s})$ & $50 \times 50 \mathrm{~mm}$ & $\$ 65.70$ & $\begin{array}{l}99.9 \% \text { pure, } \\
0.25 \mathrm{~mm} \text { foil }\end{array}$ \\
\hline Copper Tape & $6.3 \times 16.46 \mathrm{~mm}$ & $\$ 18.95$ & $3 \mathrm{M}^{\mathrm{TM}} 1181$ \\
\hline $\begin{array}{c}\text { Chromatography } \\
\text { Paper }\end{array}$ & $20 \times 20 \mathrm{~cm}$ & $\$ 57.60$ & $\begin{array}{c}\text { Whatman No. } 1 \text {, } \\
100 \text { sheets }\end{array}$ \\
\hline Transfer Tape & $\begin{array}{c}4 \text { in } x 60 \mathrm{yd} \\
2.0 \mathrm{mil}\end{array}$ & $\$ 83.78$ & $\begin{array}{c}3 \mathrm{M}^{\mathrm{TM}} 200 \mathrm{MP} \\
467 \mathrm{MP}\end{array}$ \\
\hline Deionized Water & $1.3 \mathrm{~L}$ & $\$ 53.30$ & CAS 7732-18-5 \\
\hline TechniCloth wipe & $23 \times 23 \mathrm{~cm}$ & $\$ 271.53$ & TX 609, 300 sheets \\
\hline Silver acetate & $5 \mathrm{~g}$ & $\$ 42.10$ & ReagentPlus®, 99\% \\
\hline Formic Acid & $100 \mathrm{ml}$ & $\$ 49.20$ & ACS Reagent, $\geq 88 \%$ \\
\hline $\begin{array}{l}\text { Ammonium } \\
\text { hydroxide }\end{array}$ & $500 \mathrm{ml}$ & $\$ 41.30$ & $\begin{array}{l}\text { ACS Reagent, } \\
29 \% \mathrm{NH}_{3} \text { basis }\end{array}$ \\
\hline
\end{tabular}


Appendix C

Table 4. Change in resistivity during folding

\begin{tabular}{c||c|c|c|c|c||}
\multicolumn{1}{l||}{} & \multicolumn{5}{c||}{ Fold Angle, $\theta$} \\
& $-180^{\circ}$ & $-90^{\circ}$ & $0^{\circ}$ & $90^{\circ}$ & $180^{\circ}$ \\
\hline Sample 1 & $3.75^{*}$ & 4.25 & 1 & 4.75 & 6.50 \\
\hline Sample 2 & 0.27 & 0.36 & 1 & 1.27 & 3.63 \\
\hline Sample 3 & 1.50 & 1.25 & 1 & 3.00 & 3.50 \\
\hline Sample 4 & 2.00 & 1.40 & 1 & 18.4 & 6.20 \\
\hline Sample 5 & 2.10 & 1.22 & 1 & 9.3 & 5.55 \\
\hline Mean, $\mu$ & 1.92 & 1.69 & 1 & 7.35 & 5.08 \\
\hline Standard & 1.25 & 1.48 & 0 & 6.87 & 1.42 \\
Deviation, $\sigma$ & & & & &
\end{tabular}

*Values are represented as relative resistivity, $\mathrm{R}_{\text {measured }} / \mathrm{R}_{0}$, and are unit-less. 


\section{Appendix D}

Table 5. Resistivity using two-factor sinter matrix

\begin{tabular}{|c|c|c|c|c|}
\hline Ink & \multicolumn{4}{|c|}{ Sinter* time (minutes) } \\
\hline $\begin{array}{l}\text { Concentration } \\
\qquad\left(\mu \mathrm{L} \cdot \mathrm{mm}^{-3}\right)\end{array}$ & 15 & 30 & 60 & 90 \\
\hline 0.08 & $3750 \Omega \cdot \mathrm{m}$ & $3750 \Omega \cdot \mathrm{m}$ & $3750 \Omega \cdot \mathrm{m}$ & $3750 \Omega \cdot \mathrm{m}$ \\
\hline 0.17 & $1500 \Omega \cdot \mathrm{m}$ & $750 \Omega \cdot \mathrm{m}$ & $750 \Omega \cdot \mathrm{m}$ & $525 \Omega \cdot \mathrm{m}$ \\
\hline 0.25 & $119.25 \Omega \cdot \mathrm{m}$ & $90 \Omega \cdot m$ & - & - \\
\hline 0.33 & - & $93.75 \Omega \cdot \mathrm{m}$ & $47.36 \Omega \cdot \mathrm{m}$ & \\
\hline 0.50 & $8.25 \Omega \cdot \mathrm{m}$ & $3.75 \Omega \cdot \mathrm{m}$ & - & 0.0375 \\
\hline 0.58 & - & $0.1875 \Omega \cdot \mathrm{m}$ & $0.0375 \Omega \cdot \mathrm{m}$ & $0.0375 \Omega \cdot \mathrm{m}$ \\
\hline 0.66 & $1.8 \Omega \cdot \mathrm{m}$ & $0.0375 \Omega \cdot \mathrm{m}$ & $0.0375 \Omega \cdot \mathrm{m}$ & $0.0375 \Omega \cdot \mathrm{m}$ \\
\hline 0.83 & $0.0375 \Omega \cdot \mathrm{m}$ & $0.0375 \Omega \cdot \mathrm{m}$ & $0.0375 \Omega \cdot \mathrm{m}$ & $0.0375 \Omega \cdot \mathrm{m}$ \\
\hline
\end{tabular}

*Traces were sintered using $150^{\circ} \mathrm{C}$ isothermal hold profile. It must be noted that empty cells in the matrix correspond to data points that were omitted due to either unstable readings, compromised deposition, or sample damage. 
Appendix E

Table 6. Resistivity using three-factor sinter matrix

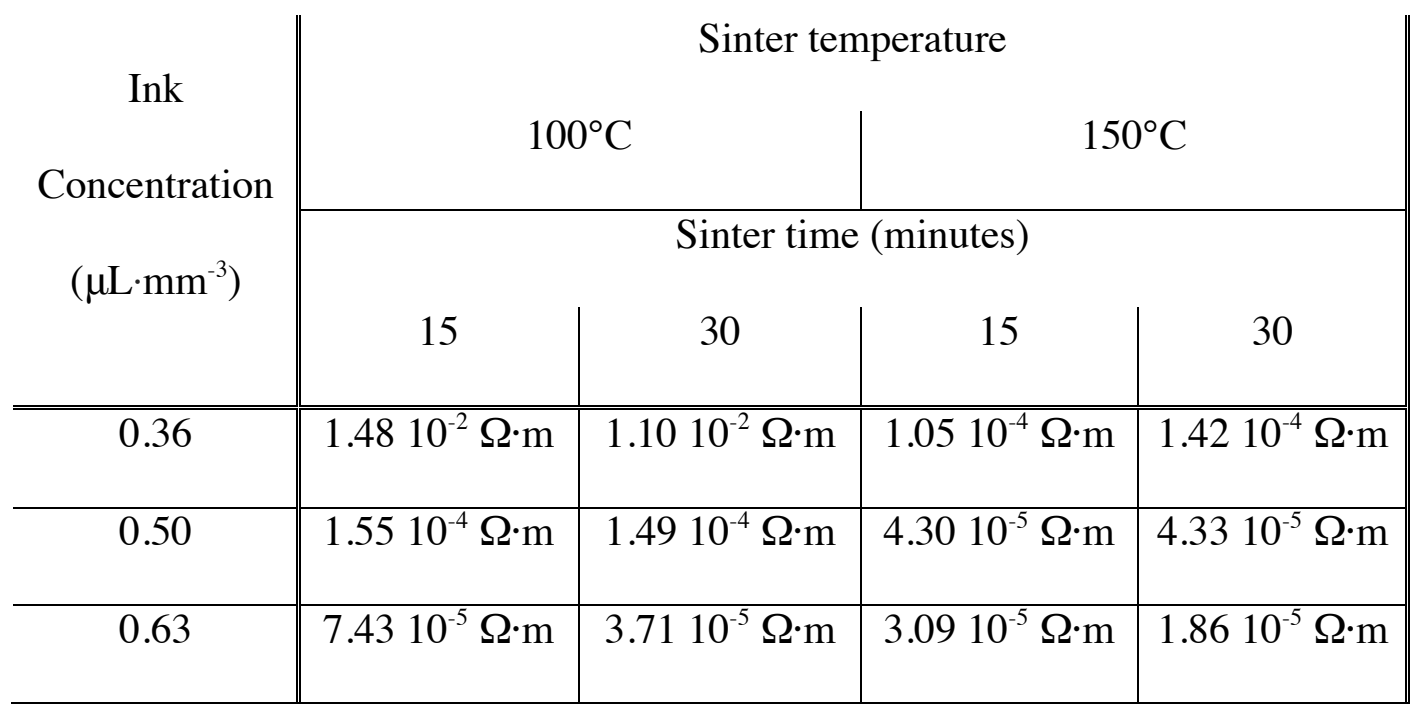


Appendix F

Table 7. Short-circuit current measurements

\begin{tabular}{|c|c|c|c|c|c|c|c|}
\hline \multirow{2}{*}{$\begin{array}{l}\text { Time } \\
\text { (min) }\end{array}$} & \multicolumn{3}{|c|}{ Current $(\mu \mathrm{A})$} & \multirow{2}{*}{$\begin{array}{l}\text { Time } \\
\text { (min) }\end{array}$} & \multicolumn{3}{|c|}{ Current $(\mu \mathrm{A})$} \\
\hline & Trial 1 & Trial 2 & Trial 3 & & Trial 1 & Trial 2 & Trial 3 \\
\hline 0 & - & 0.1 & 2.4 & 24 & 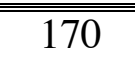 & 131 & 142 \\
\hline 1 & - & 0.2 & 3.2 & 25 & 160 & 139 & 137 \\
\hline 2 & - & 0.2 & 12.3 & 26 & 151 & 124 & 128 \\
\hline 3 & - & 0.6 & 71.8 & 27 & 144 & 124 & 123 \\
\hline 4 & - & 218 & 175.7 & 28 & 136 & 118 & 123 \\
\hline 5 & - & 302 & 372 & 29 & 130 & 122 & 117 \\
\hline 6 & - & 295 & 468 & 30 & 125 & 116 & 113 \\
\hline 7 & - & 293 & 516 & 31 & 117 & 106 & 111 \\
\hline 8 & 410 & 320 & 515 & 32 & 113 & 104 & 107 \\
\hline 9 & 510 & 307 & 467 & 33 & 110 & 99 & 102 \\
\hline 10 & 638 & 288 & 359 & 34 & 110 & 92 & 100 \\
\hline 11 & 665 & 304 & 312 & 35 & 107 & 95 & 98 \\
\hline 12 & 695 & 269 & 282 & 36 & 105 & 95 & 85 \\
\hline 13 & 728 & 250 & 248 & 37 & 102 & 94 & 80 \\
\hline 14 & 687 & 241 & 233 & 38 & 99 & 88 & 80 \\
\hline 15 & 611 & 220 & 220 & 39 & 96 & 91 & 80 \\
\hline 16 & 566 & 223 & 199 & 40 & 95 & 90 & 72 \\
\hline 17 & 511 & 205 & 185 & 41 & 91 & 86 & 75 \\
\hline 18 & 441 & 156 & 196 & 42 & 90 & 78 & 71 \\
\hline 19 & 394 & 168 & 193 & 43 & 87 & 79 & 69 \\
\hline 20 & 329 & 163 & 179 & 44 & 85 & 82 & 74 \\
\hline 21 & 265 & 157 & 169 & 45 & 84 & 81 & 68 \\
\hline 22 & 232 & 147 & 160 & & & & \\
\hline 23 & 186 & 144 & 154 & & & & \\
\hline
\end{tabular}


Appendix G

Table 8. Open-circuit voltage measurements

\begin{tabular}{|c|c|c|c|c|c|}
\hline \multirow{2}{*}{ Time (min) } & \multicolumn{2}{|c|}{ Voltage (V) } & \multirow{2}{*}{ Time (min) } & \multicolumn{2}{|c|}{ Voltage (V) } \\
\hline & Trial A & Trial B & & Trial A & Trial B \\
\hline 0 & 1.65 & 2.18 & 26 & 1.21 & 2.49 \\
\hline 1 & 1.58 & 2.05 & 27 & 1.21 & 2.48 \\
\hline 2 & 1.65 & 2.2 & 28 & 1.22 & 2.49 \\
\hline 3 & 1.91 & 2.5 & 29 & 1.25 & 2.49 \\
\hline 4 & 2.06 & 2.5 & 30 & 1.24 & 2.48 \\
\hline 5 & 2.09 & 2.49 & 31 & 1.24 & 2.48 \\
\hline 6 & 2.17 & 2.51 & 32 & 1.25 & 2.48 \\
\hline 7 & 2.26 & 2.5 & 33 & 1.27 & 2.49 \\
\hline 8 & 2.28 & 2.5 & 34 & 1.27 & 2.48 \\
\hline 9 & 2.02 & 2.49 & 35 & 1.28 & 2.48 \\
\hline 10 & 1.6 & 2.49 & 36 & 1.28 & 2.48 \\
\hline 11 & 1.36 & 2.49 & 37 & 1.27 & 2.46 \\
\hline 12 & 1.27 & 2.48 & 38 & 1.29 & 2.47 \\
\hline 13 & 1.25 & 2.47 & 39 & 1.29 & 2.48 \\
\hline 14 & 1.22 & 2.5 & 40 & 1.28 & 2.48 \\
\hline 15 & 1.2 & 2.51 & 41 & 1.28 & 2.48 \\
\hline 16 & 1.19 & 2.51 & 42 & 1.28 & 2.47 \\
\hline 17 & 1.2 & 2.49 & 43 & 1.31 & 2.47 \\
\hline 18 & 1.2 & 2.48 & 44 & 1.34 & 2.45 \\
\hline 19 & 1.19 & 2.48 & 45 & 1.3 & 2.46 \\
\hline 20 & 1.18 & 2.49 & - & - & - \\
\hline 21 & 1.17 & 2.48 & 50 & 1.23 & 2.36 \\
\hline 22 & 1.17 & 2.46 & 60 & 1.44 & 2.29 \\
\hline 23 & 1.17 & 2.48 & 70 & 1.48 & 2.26 \\
\hline 24 & 1.17 & 2.48 & & & \\
\hline 25 & 1.21 & 2.5 & & & \\
\hline
\end{tabular}

